\title{
On a completely non-unitary contraction and associated dissipative difference operator
}

\author{
Ekin Uğurlua,*, Dumitru Baleanu ${ }^{\mathrm{a}, \mathrm{b}}$ \\ ${ }^{a}$ Cankaya University, Faculty of Arts and Science, Department of Mathematics, 06530 Balgat, Ankara, Turkey. \\ ${ }^{b}$ Institute of Space Sciences, Magurele-Bucharest, Romania.
}

Communicated by A. Atangana

\begin{abstract}
In this paper, we investigate the spectral properties of dissipative difference operator, dissipative sum operator and contractive operator. Using Solomyak's method, we construct the characteristic function of the dissipative difference operator. For this purpose, we use boundary spaces and functional embeddings. Then we pass to the characteristic function of the Cayley transform of the dissipative difference operator which is a completely non-unitary contraction belonging to the class $\mathrm{C}_{0}$. With the aid of this characteristic function we achieve to pass to the minimal function of the contraction and we investigate the complete spectral analysis of both the contractive and dissipative operators. Embedding the associated contraction to its natural unitary colligation, we obtain a Carathéodory function. Moreover, self-adjoint dilation of the maximal dissipative difference operator and its incoming and outgoing eigenfunctions are constructed. Finally, the truncated CMV matrix is established which is unitary equivalent to the contractive operator. (C)2017 All rights reserved.
\end{abstract}

Keywords: Difference operator, completely non-unitary contraction, dissipative operator, characteristic function, CMV matrix. 2010 MSC: 47B361, 47B44, 47A48, 34B20, 62M15.

\section{Introduction}

A model of an operator is generally defined as an operator which is equivalent to the first one in a certain sense. These models can be described by similarity, quasi-similarity, and unitary equivalence. Unitary equivalence is particularly interested by the authors in recent years. Namely, a famous model operator of contractive operators acting on the Hilbert spaces belongs to Nagy and Foiass [16,17]. In the basic of this theory, unitary dilation of a given contraction plays an important role. An operator $\mathcal{U}$ acting on a Hilbert space $\mathcal{H}$ is called dilation of an operator $\mathrm{T}$ acting on another Hilbert space $\mathrm{H}$ such that $\mathrm{H} \subset \mathcal{H}$ if

$$
\mathrm{T}^{\mathrm{n}}=\left\{\mathrm{P}_{\mathrm{H}} \mathrm{U}^{\mathrm{n}} \mid \mathrm{H}, \mathrm{n} \geqslant 1\right\},
$$

where $\mathrm{P}_{\mathrm{H}}$ is the orthogonal projection of $\mathcal{H}$ onto $\mathrm{H}$ and $\mathcal{H}$ is called the dilation space. The geometric structure of the dilation space was given by Sarason [22]: $\mathcal{U}: \mathcal{H} \rightarrow \mathcal{H}$ is a dilation of $\mathrm{T}: \mathrm{H} \rightarrow \mathrm{H}$ if and only if

$$
\mathcal{H}=\mathrm{G}_{*} \oplus \mathrm{H} \oplus \mathrm{G},
$$

\footnotetext{
*Corresponding author

Email addresses: ekinugurlu@cankaya .edu.tr (Ekin Uğurlu), dumitru@cankaya.edu.tr (Dumitru Baleanu)
} 
where $U G \subset G, U^{*} G_{*} \subset G_{*}$ and $P_{H} U \mid H=T$. $G$ and $G_{*}$ are called the outgoing subspace and incoming subspace, respectively. If $\mathcal{U}$ is unitary on $\mathcal{H}$, then $\mathcal{U}$ is called unitary dilation. Moreover in the case that

$$
\operatorname{span}\left(\mathcal{U}^{\mathrm{n}} \mathrm{H}: \mathrm{n} \in \mathbb{Z}\right)=\mathcal{H},
$$

$\mathcal{U}$ is called minimal. Then Wold-Kolmogorov lemma [19] implies that if $\mathcal{U}: \mathcal{H} \rightarrow \mathcal{H}, \mathcal{H}=\mathrm{G}_{*} \oplus \mathrm{H} \oplus \mathrm{G}$, is the minimal unitary dilation of a contraction $\mathrm{T}: \mathrm{H} \rightarrow \mathrm{H}$ then

$$
\mathrm{G}=\bigoplus_{\mathrm{n} \geqslant 0} \mathcal{U}^{\mathrm{n}} \mathrm{E}, \mathrm{G}_{*}=\bigoplus_{\mathrm{n} \geqslant 0} \mathcal{U}^{* \mathrm{n}} \mathrm{E}_{*},
$$

where $\mathrm{E}=\mathrm{G} \ominus \mathcal{U} \mathrm{G}$ and $\mathrm{E}_{*}=\mathrm{G}_{*} \ominus \mathcal{U}^{*} \mathrm{G}_{*}$. This representation makes one possible to give more precise description of the minimal unitary dilation $\mathcal{U}$ on $\mathcal{H}=\mathrm{G}_{*} \oplus \mathrm{H} \oplus \mathrm{G}$ as

$$
\mathcal{U}=\left[\begin{array}{ccc}
\mathrm{P}_{\mathrm{G}_{*}} \mathrm{U} \mid \mathrm{G}_{*} & 0 & 0 \\
\mathrm{D}_{\mathrm{T} *} \mathrm{~V}_{*}^{*} & \mathrm{~T} & 0 \\
-\mathrm{VT}^{*} \mathrm{~V}_{*}^{*} & \mathrm{VD}_{\mathrm{T}} & \mathcal{U} \mid \mathrm{G}
\end{array}\right],
$$

where $D_{T^{*}}=\left(I-T T^{*}\right)^{1 / 2}, D_{T}=\left(I-T^{*} T\right)^{1 / 2}, V$ is a partial isometry with initial space $\overline{D_{T} H}$ and final space $E$ and $V_{*}$ is a partial isometry with initial space $\overline{D_{T^{*}} \mathrm{H}}$ and final space $E_{*}$. Note that an operator $\mathrm{V}: \mathrm{H} \rightarrow \mathrm{K}$ is called partial isometry if $\mathrm{H}=\mathrm{H}_{i} \oplus \mathrm{H}_{0}$, where $\mathrm{V}: \mathrm{H}_{i} \rightarrow \mathrm{H}$ is an isometry and $\mathrm{V} \mid \mathrm{H}_{0}=0 . \mathrm{H}_{i}$ is called the initial space of $\mathrm{V}$ and its range $\mathrm{VH}_{i}=\mathrm{VH}$ the final space of $\mathrm{V}$.

Let $\mathrm{E}$ and $\mathrm{E}_{*}$ be two Hilbert spaces such that

$$
\operatorname{dim} E=\operatorname{dim} G \ominus U G_{,} \operatorname{dim} E_{*}=\operatorname{dim} G_{*} \ominus \mathcal{U}^{*} G_{*} .
$$

Then there exists unitary mappings

$$
v: E \rightarrow G \ominus U G, v_{*}: E_{*} \rightarrow G_{*} \ominus \mathcal{U}^{*} \mathrm{G}
$$

that identify these spaces. Therefore with the following unitary mappings called functional embeddings,

$$
\begin{array}{rlrl}
\pi: L^{2}(E) & \rightarrow \bigoplus_{n \in \mathbb{Z}} \mathcal{U}^{\mathrm{n}}(\mathrm{G} \ominus \mathcal{U G}), & \pi_{*}: \mathrm{L}^{2}\left(\mathrm{E}_{*}\right) & \rightarrow \bigoplus_{n \in \mathbb{Z}} \mathcal{U}^{\mathrm{n}}\left(\mathrm{G}_{*} \ominus \mathcal{U}^{*} \mathrm{G}_{*}\right), \\
\sum_{n} z^{\mathrm{n}} e_{\mathrm{n}} \rightarrow \sum_{n} \mathcal{U}^{\mathrm{n}} v e_{\mathrm{n}}, & \sum_{n} z^{n} e_{n}^{*} \rightarrow \sum_{n} \mathcal{U}^{\mathrm{n}+1} v_{*} e_{n}^{*},
\end{array}
$$

where $e_{\mathrm{n}} \in \mathrm{E}$ and $e_{\mathrm{n}}^{*} \in \mathrm{E}_{*}$, one can define the functional mappings as

$$
\prod: \mathrm{L}^{2}(\mathrm{E}) \oplus \mathrm{L}^{2}\left(\mathrm{E}_{*}\right) \rightarrow \mathcal{H}, \quad(\mathrm{f}, \mathrm{g}) \rightarrow \pi \mathrm{f}+\pi_{*} \mathrm{~g} .
$$

Finally letting

$$
\Theta f=\pi_{*}^{*} \pi f, f \in L^{2}(E),
$$

it is obtained the characteristic function $\Theta$ of the contraction T. An explicit form of the characteristic function $\Theta$ is given by

$$
\Theta_{\mathrm{T}}(z) h=\mathrm{V}_{*}\left(-\mathrm{T}+z \mathrm{D}_{\mathrm{T} *}\left(\mathrm{I}-z \mathrm{~T}^{*}\right)^{-1} \mathrm{D}_{\mathrm{T}}\right) \mathrm{V}^{*} \mathrm{~h}, \mathrm{~h} \in \mathrm{E},
$$

where

$$
\mathrm{V}: \mathfrak{D}_{\mathrm{T}} \leftrightarrow \mathrm{E}, \mathrm{V}_{*}: \mathfrak{D}_{\mathrm{T}^{*}} \leftrightarrow \mathrm{E}_{*},
$$

$\mathfrak{D}_{\mathrm{T}}=\overline{\mathrm{D}_{\mathrm{T}} \mathrm{H}}$ and $\mathfrak{D}_{\mathrm{T}^{*}}=\overline{\mathrm{D}_{\mathrm{T}^{*}} \mathrm{H}}$. If one chooses $\mathrm{E}=\mathfrak{D}_{\mathrm{T}}$ and $\mathrm{E}_{*}=\mathfrak{D}_{\mathrm{T}^{*}}$, then $\Theta_{\mathrm{T}}$ is reduced to

$$
\Theta_{\mathrm{T}}(z)=-\mathrm{T}+z \mathrm{D}_{\mathrm{T} *}\left(\mathrm{I}-z \mathrm{~T}^{*}\right)^{-1} \mathrm{D}_{\mathrm{T}}
$$

which is the well-known characteristic function of Nagy and Foiaş. 
Solomyak used this coordinate free approach to obtain the characteristic function of a completely non-self-adjoint (simple) maximal dissipative operator [26]. In fact, using boundary spaces of the dissipative operator, Solomyak constructed isometric isomorphism such that the characteristic function of the maximal dissipative operator $A$ and its Cayley transform $T$ is connected by

$$
S_{A}(\lambda)=\Theta_{\mathrm{T}}\left(\frac{\lambda-i}{\lambda+i}\right), \quad \text { and } \quad S_{A}(\lambda)=\mathcal{P}_{*}\left(A^{*}-\lambda I\right)^{-1}(A-\lambda I) \mathcal{P}^{-1},
$$

where $\mathcal{P}$ and $\mathcal{P}_{*}$ are natural projections. Solomyak achieved to introduce a self-adjoint dilation of the maximal dissipative operator with the aid of free parameters. Moreover, using the characteristic function of the maximal dissipative operator, Solomyak described directly the generalized eigenfunctions of the selfadjoint dilation. We use this construction in Sections 3 and 4.

In this paper, using the connection of the characteristic functions of a maximal dissipative operator and its Cayley transform we investigate the complete spectral analysis of a maximal dissipative difference operator. Namely, passing to the Cayley transform of the maximal dissipative difference operator we obtain a contraction defined on a Hilbert space. Moreover, we obtain that this contraction is a completely non-unitary contraction (c.n.u.) belonging to the class $\mathrm{C}_{0}$. The spectral properties of the c.n.u. contractions of the class $C_{0}$ has been investigated by Nagy and Foias [16, 17]. It is known that for every contraction $\mathrm{T} \in \mathrm{C}_{0}$ there exists a minimal function $\mathrm{m}_{\mathrm{T}}$ which is an inner function belonging to the class $\mathrm{H}^{\infty}\left(\mathrm{H}^{p}\right.$ is the Hardy class). The minimal function $m_{T}$ gives some information about the spectral properties of a contraction $T$. Indeed, the spectrum of the contraction $T \in \mathrm{C}_{0}$ and the zeros of the minimal function $\mathrm{m}_{\mathrm{T}}$ in the open disc $\mathbf{D}$ and of the complement, in the unit circle $\mathbf{C}$, of the union of the arcs of $\mathbf{C}$ on which $m_{\mathrm{T}}$ is analytic, coincide with each other. Furthermore, in the interior of the unit circle $\mathbf{C}$, the points of the spectrum are eigenvalues of $T$. As a characteristic value of $T, \lambda$ has finite index, equal to its multiplicity as a zero of $m_{\mathrm{T}}$. Finally, completeness of the root functions of $T$ associated with the points of the spectrum of $\mathrm{T}$ in $\mathbf{D}$ can be obtained as proving that the minimal function $\mathrm{m}_{\mathrm{T}}$ is a Blaschke product. On the other side, there is a nice connection between the minimal function $m_{T}$ and the characteristic function $\Theta_{\mathrm{T}}$. Therefore, this connection allows us to investigate the complete spectral analysis of a contractive operator and hence of a dissipative operator. We should note that we will also use the resolvent operator of the dissipative difference operator.

In the literature there are some methods to investigate the spectral properties of a dissipative operator acting on a Hilbert space. For example, Livšic's theorem, Lidskiî's theorem, Krein's theorem and Pavlov's method are used for such operators [12, 21]. In particular, Pavlov's method is based on the fact that there is a connection between a continuous semigroup of contractions $\{Z(t)\}_{t \geqslant 0}$ and its cogenerator $Z$. Therefore every model of $Z$ generates a model of $\{Z(t)\}_{t \geqslant 0}$. In this paper, we do not use this connection. In fact, we obtain the characteristic function of the dissipative operator directly and hence we are able to pass to the characteristic function of the contractive operator. Then we investigate the spectral analysis of both of them.

It is known that Sz.-Nagy-Foiaş characteristic function theory is a special case of unitary colligation theory. To be more precise, we should note that a contraction with its defect operators can be embedded into a unitary colligation $[5,8]$. Arlinskii et al. studied the relation of c.n.u. contractions with rank-one defects and corresponding unitary colligations [5]. Hence using the results obtained in [5] we introduce some theorems about the Cayley transform of the dissipative difference operator.

Jacobi matrices are useful to understand the characterization of self-adjoint, non-self-adjoint, and unitary operators acting on separable Hilbert spaces. Indeed, multiplication operators on the Hilbert spaces $L^{2}(\mathbb{R})$ or $L^{2}(\mathbf{C})$ associated with the probability measure $m$ on the real line $\mathbb{R}$ or on the unit circle C, respectively, is unitary equivalent to the self-adjoint or unitary operators with a simple spectrum acting on some Hilbert spaces [2]. Tri-diagonal Jacobi matrix representation of self-adjoint operators with simple spectrum was introduced by Stone [1]. The non-self-adjoint version of Stone's theorem has been introduced by Arlinskiı̌ and Tsekanovskiı̌ [6]. Moreover, the canonical matrix representation of unitary operators with simple spectrum has been introduced by Cantero et al. [9] with the help of five-diagonal 
unitary matrices called CMV matrices. Arlinskiǔ et al. [5] obtained a connection between truncated CMV matrix and Sz.-Nagy-Foiaş characteristic function. Therefore, we introduce truncated CMV matrix associated with the Cayley transform.

This paper is organized as follows. In Section 2, we introduce the difference dissipative operator and its inverse operator. Using compactness property with a finite-rank imaginary component we obtain some spectral results. In Section 3, we construct a contractive operator which is c.n.u. of the class $\mathrm{C}_{0}$. Using the characteristic function of the maximal dissipative difference operator we introduce some results. In Section 4, we establish self-adjoint dilation of the maximal dissipative difference operator and incoming and outgoing eigenfunctions of the dilation, directly. In Section 5, we introduce several properties of the contraction Cayley transform of the maximal dissipative difference operator. Indeed, multiplicity of the Cayley transform and its adjoint operator, the characteristic function of the unitary colligation with basic operator and truncated CMV matrix which is unitary equivalent to the Cayley transform are obtained.

Throughout the paper the notations $\mathbf{C}$ and $\mathbf{D}$ will be used to denote the unit circle $\mathbf{C}=\{\mu \in \mathbb{C}:|\mu|=1\}$ and unit disc $\mathbf{D}=\{\mu \in \mathbb{C}:|\mu|<1\}$.

\section{Infinite difference equations}

We denote by $\ell^{2}(\mathbb{Z})$, where $\mathbb{Z}:=\{\cdots,-2,-1,0,1,2, \cdots\}$, the Hilbert space consisting of all sequences $y=\left\{y_{n}\right\}_{-\infty}^{\infty}$ such that

$$
\sum_{n=-\infty}^{\infty}\left|y_{n}\right|^{2}<\infty
$$

with the inner product

$$
(y, x)=\sum_{n=-\infty}^{\infty} y_{n} \bar{x}_{n} .
$$

We consider the following second order difference equations

$$
J y=\left\{(J y)_{n}\right\}_{-\infty}^{\infty}:=a_{n-1} y_{n-1}+b_{n} y_{n}+a_{n} y_{n+1}=\lambda y_{n}, n \in \mathbb{Z},
$$

where $y=\left\{y_{n}\right\}_{-\infty}^{\infty}$ and $\lambda$ is the complex parameter.

Let $M$ be a subspace of $\ell^{2}(\mathbb{Z})$ consisting of all sequences $y=\left\{y_{n}\right\}_{-\infty}^{\infty} \in \ell^{2}(\mathbb{Z})$ such that $J y \in \ell^{2}(\mathbb{Z})$. We define the operator $L$ on $M$ as $L y=J y, y \in M$.

Consider the subspace $M_{0}^{\prime}$ of $\ell^{2}(\mathbb{Z})$ consisting of all sequences $y=\left\{y_{n}\right\}_{-\infty}^{\infty} \in \ell^{2}(\mathbb{Z})$ such that $y$ has only a finite number of nonzero components. The operator $L_{0}^{\prime}$ the restriction of $L$ to $M_{0}^{\prime}$ is symmetric. Therefore it admits closure. We denote the closure of $\mathrm{L}_{0}^{\prime}$ by $\mathrm{L}_{0}$. The operator $\mathrm{L}_{0}$ is a closed, symmetric operator with equal deficiency indices $(r, r)$, where $r=0,1$, 2 . Moreover, it is obtained that $L_{0}^{*}=L$ [23].

For arbitrary two sequences $y=\left\{y_{n}\right\}_{-\infty}^{\infty}, x=\left\{x_{n}\right\}_{-\infty}^{\infty} \in M$, the Green's formula is obtained as

$$
\sum_{n=-\infty}^{\infty}\left\{(L y)_{n} x_{n}-y_{n}(L x)_{n}\right\}=[y, x](\infty)-[y, x](-\infty)
$$

where

$$
[y, x](n)=a_{n}\left(y_{n+1} x_{n}-y_{n} x_{n+1}\right), n \in \mathbb{Z} .
$$

In fact, to obtain the equality (2.2), it is sufficient to consider the finite sum and then pass to the infinity as well as negative infinity. (2.2) particularly implies for arbitrary two sequences $y=\left\{y_{n}\right\}_{-\infty}^{\infty}, \chi=\left\{\chi_{n}\right\}_{-\infty}^{\infty} \in$ $M$ that the values $[y, \chi]( \pm \infty)$ and $[y, \bar{\chi}]( \pm \infty)$ exist and are finite. The latter one follows from the fact that $a_{n}$ and $b_{n}$ are real numbers.

Equation (2.3) can be regarded as the Wronskian of the solutions $y$ and $\chi$ of $(2.1)$ as $W[y, \chi](n)=$ $[y, \chi](n)$. 
We consider the solutions $u$ and $z$ such that $u=\left\{u_{n}\right\}_{-\infty}^{\infty}, z=\left\{z_{n}\right\}_{-\infty}^{\infty}$ of $J y=0$ satisfying

$$
[\mathrm{u}, z] \equiv 1, \mathrm{n} \in \mathbb{Z}
$$

In this paper, we assume that the deficiency indices of $L_{0}$ are $(2,2)$. In other words, we assume that $L_{0}$ is in Weyl-Hamburger limit-circle case. Therefore, $u$ and $z$ also belong to $M$.

Using (2.4) it is obtained for arbitrary two functions $y, x \in M$ that

$$
[y, \chi](n)=[y, u](n)[\chi, z](n)-[y, z](n)[\chi, u](n), n \in \mathbb{Z} .
$$

$M_{0}$ can be described with the aid of real solutions $u$ and $z$. Indeed, $M_{0}$ consists of those functions $y \in M$ such that

$$
[y, u](\infty)=[y, z](\infty)=[y, u](-\infty)=[y, z](-\infty)=0
$$

For $y \in M$, we consider the following boundary value problem

$$
\mathrm{Jy}=\lambda y, \quad[y, u](-\infty)-l[y, z](-\infty)=0, \quad[y, u](\infty)-h[y, z](\infty)=0,
$$

where $l$ is a real number, $h$ is a complex number such that $h=\operatorname{Reh}+i \operatorname{Imh}$ with $\operatorname{Imh}>0$.

We define the operator $\mathcal{J}$ on $M_{\mathcal{J}}$ as $\mathcal{J} y=J y$, where $M_{\mathcal{J}}$ is the domain of $\mathcal{J}$ consisting of all functions $y \in M$ satisfying $[y, u](-\infty)-l[y, z](-\infty)=0,[y, u](\infty)-h[y, z](\infty)=0$, Imh $>0$. Therefore the equation

$$
\partial y=\lambda y, y \in M_{\mathcal{J}}
$$

coincides with the problem (2.6).

The adjoint operator $\mathcal{J}^{*}$ of $\mathcal{J}$ is defined by $\mathcal{J}^{*} y=J y, y \in M_{\mathcal{J}^{*}}$, where $M_{\mathcal{J}^{*}}$ is the domain of $\mathcal{J}^{*}$ consisting of all functions $y \in M$ satisfying $[y, u](-\infty)-l[y, z](-\infty)=0,[y, u](\infty)-\bar{h}[y, z](\infty)=0, \operatorname{Imh}>0$.

We shall remind that an operator $T$ in a Hilbert space $H$ with the domain $D(T)$ is said to be dissipative if

$$
\operatorname{Im}(T y, y) \geqslant 0, y \in D(T)
$$

Theorem 2.1. $J$ is dissipative in $\ell^{2}(\mathbb{Z})$.

Proof. For arbitrary function $y=\left\{y_{n}\right\}_{-\infty}^{\infty} \in M_{\mathcal{J}}$ we obtain with a direct calculation that

$$
(J y, y)-(y, \partial y)=[y, \bar{y}](\infty)-[y, \bar{y}](-\infty)
$$

Moreover using (2.5) one gets

$$
[y, \bar{y}](\infty)=[y, u](\infty)[\bar{y}, z](\infty)-[y, z](\infty)[\bar{y}, u](\infty)
$$

Since $y \in M_{\mathcal{J}}$ we have from (2.8) that

$$
[y, \bar{y}](\infty)=2 i \operatorname{Imh}|[y, z](\infty)|^{2},[y, \bar{y}](-\infty)=0 .
$$

Equations (2.7) and (2.9) give

$$
\operatorname{Im}(\partial y, y)=\operatorname{Imh}|[y, z](\infty)|^{2}
$$

Therefore (2.10) completes the proof.

Definition 2.2. A dissipative operator $A$ is called maximal dissipative if it does not have a proper dissipative extension.

Maximal dissipative extensions given for a minimal symmetric operator with equal deficiency indices can be obtained by Gorbachuks' extension theorem [13]. The proof of the next theorem can be obtained from [3, p. 111]. 
Theorem 2.3. $J$ is maximal dissipative in $\ell^{2}(\mathbb{Z})$.

Definition 2.4. A non-self-adjoint operator $A$ acting on a Hilbert space $H$ is called simple if there is no invariant subspace of $\mathrm{H}$ on which $A$ has self-adjoint part there.

Theorem 2.5. $J$ is a simple dissipative operator in $\ell^{2}(\mathbb{Z})$.

Proof. Consider that $\mathcal{J}$ has a self-adjoint part $\mathcal{J}_{s}$ with domain $M_{\mathcal{J}_{s}}$ on a nontrivial subspace of $\ell^{2}(\mathbb{Z})$. Then for $y \in M_{\jmath_{s}}$ we have

$$
0=\left(\mathfrak{J}_{s} y, y\right)-\left(y, J_{s} y\right)=2 i \operatorname{Imh}|[y, z](\infty)|^{2} .
$$

Therefore from $(2.11)$ we obtain $[y, z](\infty)=0$. Substituting this in $(2.6)$ we get that $[y, u](\infty)=0$. Let $y=Q(\lambda)$ and $\widetilde{Q}(\lambda)$ be the solutions of $(2.1)$ such that

$$
[\mathrm{Q}, \widetilde{\mathrm{Q}}]=1 \text {. }
$$

Then using (2.12) and (2.5) we obtain

$$
1=[\mathrm{Q}, \widetilde{\mathrm{Q}}](\infty)=[\mathrm{Q}, \mathrm{u}](\infty)[\widetilde{\mathrm{Q}}, z](\infty)-[\mathrm{Q}, z](\infty)[\widetilde{\mathrm{Q}}, \mathrm{u}](\infty)=0 .
$$

This contradiction completes the proof.

Corollary 2.6. All eigenvalues of $\mathcal{J}$ lie in the open upper half-plane. In particular, zero is not an eigenvalue of $\mathcal{J}$.

Let $P(\lambda)$ be the solution of (2.1) satisfying the conditions

$$
[P(\lambda), u](-\infty)=l, \quad[P(\lambda), z](-\infty)=1 .
$$

Consider the function

$$
\omega_{h}(\lambda)=[P(\lambda), u](\infty)-h[P(\lambda), z](\infty) .
$$

The zeros of $\omega_{h}$ coincide with the eigenvalues of $\mathcal{\partial}$. Since $\omega_{h}$ is an entire function [14], all eigenvalues of $\mathcal{J}$ form a discrete subset of $\mathbb{C}$ with a possible limit point at infinity. However, more detailed analysis will be obtained with the aid of the resolvent operator and the characteristic function of $\mathcal{J}$.

Now we shall find the inverse operator of $\mathcal{J}$. For this purpose let us consider the functions $w=u-l z$ and $v=u-h z$, where $w=\left\{w_{n}\right\}_{-\infty}^{\infty}, v=\left\{v_{n}\right\}_{-\infty}^{\infty}, w_{n}=u_{n}-l z_{n}$, and $v_{n}=u_{n}-h z_{n}, n \in \mathbb{Z}$. Taking

$$
\mathrm{G}=\left\{\mathrm{G}_{\mathrm{nm}}\right\}_{n, m=-\infty}^{\infty}, \mathrm{G}_{\mathrm{nm}}=\left\{\begin{array}{l}
w_{n} v_{m},-\infty<\mathrm{n} \leqslant \mathrm{m}<\infty, \\
w_{m} v_{n},-\infty<\mathrm{m} \leqslant \mathrm{n}<\infty,
\end{array}\right.
$$

one obtains the inverse operator $\mathcal{R}$ of $\mathcal{J}$ as

$$
(G, \bar{a})=(\mathcal{R} a)_{\mathfrak{n}}=\sum_{m=-\infty}^{\infty} G_{n m} a_{m}, a=\left\{a_{n}\right\}_{-\infty}^{\infty} \in \ell^{2}(\mathbb{Z}) .
$$

Since $\mathrm{L}_{0}$ has the deficiency indices $(2,2), \mathcal{R}$ acts on the Hilbert space $\ell^{2}(\mathbb{Z})$, i.e., $\mathcal{R}: \ell^{2}(\mathbb{Z}) \rightarrow \ell^{2}(\mathbb{Z})$. Moreover, $\mathcal{R}$ is a Hilbert-Schmidt operator, because the inequality

$$
\sum_{n=-\infty}^{\infty} \sum_{m=-\infty}^{\infty}\left|G_{n m}\right|^{2}<\infty
$$

holds. $\mathcal{R}$ can be written as $\mathcal{R}=\mathcal{R}_{1}+i \mathcal{R}_{2}$, i.e., the sum of real and imaginary parts. It is known that $\mathcal{R}_{1}$ is a self-adjoint Hilbert-Shmidt operator and $\mathcal{R}_{2}$ is a finite-rank self-adjoint operator [27]. Therefore from [6] and [15] we obtain the following results. 


\section{Theorem 2.7.}

(i) Eigenvalues of $\mathcal{R}$ are countable;

(ii) zero is the only possible limit point of the eigenvalues;

(iii) zero must belong to the spectrum of $\mathcal{R}$, however, may not be an eigenvalue of $\mathcal{R}$;

(iv) the non-real spectrum of $\mathcal{R}$ consists of eigenvalues of finite algebraic multiplicities and limit points of non-real spectrum belong to the spectrum of the real part $\mathcal{R}_{1}$.

Therefore from Theorem 2.7 we obtain the following theorem.

\section{Theorem 2.8.}

(i) Eigenvalues of $\mathcal{J}$ are countable;

(ii) infinity is the only possible limit point of the eigenvalues of $\mathcal{J}$;

(iii) infinity must belong to the spectrum of $\mathcal{J}$, however, may not be an eigenvalue of $\mathcal{J}$.

The problem of completeness of root (eigen and associated) functions of $\mathcal{J}$ will be investigated by the characteristic function of $\mathcal{J}$.

\section{Characteristic functions}

In this section we will construct the characteristic function of the maximal dissipative operator $\mathcal{J}$. Then we will pass to the characteristic function of the Cayley transform of it. For this purpose, following Solomyak [26] we will use coordinate-free approach. But before the construction of the contraction we shall remind the well-known results on dissipative operators and associated contractions $[16,17,26]$.

\section{Lemma 3.1.}

(i) Assume the operator $\mathrm{L}_{0}$ is dissipative. Then the operator $\mathrm{T}_{0}=\mathrm{K}\left(\mathrm{L}_{0}\right)=\left(\mathrm{L}_{0}-\mathrm{iI}\right)\left(\mathrm{L}_{0}+\mathrm{iI}\right)^{-1}$ is a contraction from $\left(\mathrm{L}_{0}+\mathrm{iI}\right) \mathrm{D}\left(\mathrm{L}_{0}\right)$ onto $\left(\mathrm{L}_{0}-\mathrm{iI}\right) \mathrm{D}\left(\mathrm{L}_{0}\right)$ and $\mathrm{L}_{0}=\mathrm{i}\left(\mathrm{I}+\mathrm{T}_{0}\right)\left(\mathrm{I}-\mathrm{T}_{0}\right)^{-1}$. For each contraction $\mathrm{T}_{0}$ such that $1 \notin \sigma_{p}\left(T_{0}\right)$ (the point spectrum of the operator), operator $\mathrm{L}_{0}=\mathrm{K}^{-1}\left(\mathrm{~T}_{0}\right), \mathrm{D}\left(\mathrm{L}_{0}\right)=\left(\mathrm{I}-\mathrm{T}_{0}\right) \mathrm{D}\left(\mathrm{T}_{0}\right)$, is dissipative.

(ii) Each dissipative operator $\mathrm{L}_{0}$ has a maximal dissipative extension $\mathrm{L}$. A maximal dissipative operator is closed.

(iii) A maximal dissipative operator is maximal dissipative if and only if $\mathrm{T}=\mathrm{K}(\mathrm{L})$ is a contraction such that $\mathrm{D}(\mathrm{T})=\mathrm{H}$ and $1 \notin \sigma_{\mathrm{p}}(\mathrm{T})$.

(iv) If $\mathrm{L}$ is a maximal dissipative operator, $\mathrm{L}=\mathrm{K}^{-1}(\mathrm{~T})$, then $-\mathrm{L}^{*}$ is also maximal dissipative, $\mathrm{L}^{*}=-\mathrm{K}^{-1}\left(\mathrm{~T}^{*}\right)$.

(v) If $\mathrm{L}$ is a maximal dissipative operator, then $\sigma(\mathrm{T}) \subset \overline{\mathbb{C}}_{+},\left\|(\mathrm{L}-\lambda \mathrm{I})^{-1}\right\| \leqslant|\operatorname{Im} \lambda|^{-1}, \lambda \in \mathbb{C}_{-}$.

Note that in Lemma 3.1, the linear operators are considered in a separable Hilbert space $\mathrm{H}$, not necessarily bounded, $\mathrm{D}(\cdot)$ denotes the domain of the corresponding operator, $\mathbb{C}_{+}$and $\mathbb{C}_{-}$denote the upper and lower half-plane, respectively, and $\sigma(\cdot)$ is the spectrum of the corresponding operator.

Now we shall consider the Cayley transform $\mathcal{T}$ of the dissipative operator $\mathcal{J}$ as follows

$$
\mathcal{T}=(\mathcal{J}-\mathfrak{i} \mathbf{1})(\mathcal{J}+\mathfrak{i} \mathbf{1})^{-1}
$$

where 1 is the identity operator in the Hilbert space $\ell^{2}(\mathbb{Z})$. Because $\mathcal{J}$ is maximal dissipative, domain of $\mathcal{T}$ is the whole Hilbert space $\ell^{2}(\mathbb{Z})$. One immediately gets that

$$
\|\mathfrak{T}\|<1
$$

Indeed, letting $(\mathcal{J}+i \mathbf{1})^{-1} f=y, f=\left\{f_{n}\right\}_{-\infty}^{\infty}$, we have

$$
\|(\mathcal{J}-\mathbf{i} \mathbf{1}) y\|^{2}<\|(\mathcal{J}+\mathbf{i} \mathbf{1}) y\|^{2} \Leftrightarrow 2 \operatorname{Im}(\mathcal{J} y, y)>0 .
$$

It is obtained

$$
\mathcal{J}=\mathfrak{i}(\mathbf{1}+\mathcal{T})(\mathbf{1}-\mathcal{T})^{-1} .
$$


Note that 1 is not an eigenvalue of $\mathcal{T}$. However this does not imply that $\mathcal{J}$ is a bounded operator (see [17, p. 143, p. 171]).

We also have

$$
\left\|\mathcal{T}^{*}\right\|<1 .
$$

Let $D_{\mathcal{T}}=\left(\mathbf{1}-\mathcal{T}^{*} \mathcal{T}\right)^{1 / 2}$ and $D_{\mathcal{T}^{*}}=\left(\mathbf{1}-\mathcal{T T}^{*}\right)^{1 / 2}$ be the defect operators of $\mathcal{T}$ acting on $\ell^{2}(\mathbb{Z}), \mathfrak{D}_{\mathcal{T}}=$ $\overline{D_{\mathcal{T}} \ell^{2}(\mathbb{Z})}$ and $\mathfrak{D}_{\mathrm{T}^{*}}=\overline{\mathrm{D}_{\mathcal{T}^{*}} \ell^{2}(\mathbb{Z})}$ be the defect spaces and $\mathfrak{d}_{\mathcal{T}}=\operatorname{dim} \mathfrak{D}_{\mathcal{T}}$ and $\mathfrak{d}_{\mathcal{T}^{*}}=\operatorname{dim} \mathfrak{D}_{\mathcal{T}^{*}}$ be the defect indices of $\mathcal{T}$. The relations $\mathcal{T D}_{\mathcal{T}}=\mathrm{D}_{\mathcal{T}^{*}} \mathcal{T}, \mathcal{T}^{*} \mathrm{D}_{\mathcal{T}^{*}}=\mathrm{D}_{\mathcal{T}^{*}} \mathcal{T}^{*}$ hold.

Definition 3.2. A contraction $\mathrm{C}$ on a Hilbert space $\mathrm{H}$ is called c.n.u. if it has no non-zero reducing subspace $\mathcal{C}$ which $\mathrm{C} \mid \mathcal{C}$ is a unitary operator.

(3.1) implies the following theorem.

Theorem 3.3. $\mathcal{T}$ is c.n.u. contraction in $\ell^{2}(\mathbb{Z})$.

Definition 3.4 ([16-19]). The classes $C_{0}$ and $C_{.0}$ of contractions are defined as

$$
T \in C_{0} \text {. if } T^{n} f \rightarrow 0 \text { for all } f, T \in C_{.0} \text { if } T^{* n} f \rightarrow 0 \text { for all } f .
$$

Asymptotic classifications of $\mathrm{C}_{0}$, and $\mathrm{C}_{.0}$ are given as

$$
C_{0 .}=\left\{T:\|T\| \leqslant 1, \lim _{n}\left\|T^{n} f\right\|=0 \text { for every } f\right\}, \quad C_{.0}=\left\{T:\|T\| \leqslant 1, \lim _{n}\left\|T^{* n} f\right\|=0 \text { for every } f\right\} .
$$

$\mathrm{C}_{00}$ is defined as $\mathrm{C}_{00}=\mathrm{C}_{0 .} \cap \mathrm{C}_{.0}$.

Theorem 3.5. $\mathcal{T} \in \mathrm{C}_{00}$.

Proof. It is known that the following inequality holds

$$
\left\|\mathcal{T}^{n} f\right\| \leqslant\|\mathcal{T}\|^{n}\|f\| .
$$

Therefore using (3.1) in (3.3) and letting $n \rightarrow \infty$ we obtain that $\mathcal{T} \in \mathrm{C}_{.0}$. On the other side, we have

$$
\left\|\mathcal{T}^{* n} y\right\| \leqslant\left\|\mathcal{T}^{*}\right\|^{\mathrm{n}}\|\mathrm{f}\|
$$

Therefore (3.2) and (3.4) imply for $n \rightarrow \infty$ that $\mathcal{T} \in \mathrm{C}_{.0}$. Consequently the proof is completed.

To obtain the characteristic function of the contraction $\mathcal{T}$, now we shall construct the characteristic function of the dissipative operator $\mathcal{J}$. Therefore we shall use Solomyak's method [26].

Definition 3.6. Let $A$ be a maximal dissipative operator with the domain $D(A)$. The subspace

$$
\mathrm{G}_{\mathrm{A}}=\left\{\mathrm{y} \in \mathrm{D}(\mathrm{A}) \cap \mathrm{D}\left(\mathrm{A}^{*}\right): A \mathrm{y}=\mathrm{A}^{*} \mathrm{y}\right\}
$$

is called the Hermitian part of domain of $A$.

For a maximal dissipative operator $A$ and its Hermitian domain $G_{A}$, let us consider the natural projection $\mathcal{P}$ as $\mathcal{P}: D(A) \rightarrow D(A) / G_{A}$, where $D(A) / G_{A}$ is the quotient space. On the quotient space following inner product is defined

$$
\langle\mathcal{P} y, \mathcal{P} x\rangle=\frac{i}{2}((y, A x)-(A y, x)), y, x \in D(A) .
$$

The completion of the quotient space $D(A) / G_{A}$ is denoted by $F(A)$ with respect to the corresponding norm. In a similar manner it is defined $F_{*}(A):=\mathrm{F}\left(-A^{*}\right)$. Similarly, the projection $\mathcal{P}_{*}$ is defined as $\mathcal{P}_{*}: \mathrm{D}\left(A^{*}\right) \rightarrow \mathrm{D}\left(A^{*}\right) / \mathrm{G}_{A}$. Then we have

$$
\|\mathcal{P} y\|_{\mathrm{F}}^{2}=\operatorname{Im}(A y, y),\left\|\mathcal{P}_{*} y\right\|_{\mathrm{F}_{*}}^{2}=-\operatorname{Im}\left(A^{*} y, y\right) .
$$


$F(A)$ and $F_{*}(A)$ are called the boundary spaces of the operator $A$.

Note that in the case that if one has all dissipative extensions of a symmetric operator $A$, then $G_{A}$ is dense in the Hilbert space. For $y \in D(A) \cap D\left(A^{*}\right)$, one has $A y=A^{*} y$, i.e., $D(A) \cap D\left(A^{*}\right)=G_{A}$ [26].

There exist isometric isomorphisms $\rho: \mathrm{F}(\mathcal{J}) \rightarrow \mathfrak{D}_{\mathcal{T}}$ and $\rho_{*}: F_{*}(\mathcal{I}) \rightarrow \mathfrak{D}_{\mathcal{T}^{*}}$ [26] such that

$$
\rho \mathcal{P}(\mathbf{1}-\mathcal{T})=\mathrm{D}_{\mathcal{T}}, \rho_{*} \mathcal{P}_{*}\left(\mathbf{1}-\mathcal{T}^{*}\right)=\mathrm{D}_{\mathcal{T}^{*}} .
$$

A c.n.u. contraction $\mathcal{T}$ is defined to within a unitary equivalence of the characteristic function $\Theta_{\mathcal{T}}$ : $E \rightarrow E_{*}$, where $E$ and $E_{*}$ are auxiliary Hilbert spaces, isomorphic to $\mathfrak{D}_{\mathcal{T}}$ and $\mathfrak{D}_{\mathcal{T}^{*}}$, respectively. Fixing arbitrary isometric isomorphisims $\Omega: E \rightarrow \mathfrak{D}_{\mathcal{T}}$ and $\Omega_{*}: E_{*} \rightarrow \mathfrak{D}_{\mathcal{T}^{*}}$ it is obtained

$$
\Theta_{\mathcal{T}}(\mu)=\Omega_{*}^{*}\left(-\mathcal{T}+\mu D_{\mathcal{T}^{*}}\left(\mathbf{1}-\mu \mathcal{T}^{*}\right) D_{\mathcal{T}}\right) \Omega
$$

and

$$
\Theta_{\mathcal{T}}(\mu) \Omega^{*} D_{\mathcal{T}}=\Omega_{*}^{*} D_{\mathcal{T} *}\left(\mathbf{1}-\mu \mathcal{T}^{*}\right)^{-1}(\mu \mathbf{1}-\mathcal{T}) .
$$

The characteristic function $S_{A}$ of the completely non-self-adjoint maximal dissipative operator $A$ is defined on the sense set $D(A) / G_{A}$ as $S_{A}: F(A) \rightarrow F_{*}(A)$ by the equality [26]

$$
S_{A}(\lambda)=\mathcal{P}_{*}\left(A^{*}-\lambda I\right)^{-1}(A-\lambda I) \mathcal{P}^{-1} .
$$

Now we are ready to obtain the characteristic function of $\mathcal{J}$.

Theorem 3.7. The characteristic function $S_{\mathcal{J}}(\lambda)$ of the dissipative operator $\mathcal{J}$ is as follows

$$
S_{\mathfrak{J}}(\lambda)=\frac{\omega_{h}(\lambda)}{\omega_{\bar{h}}(\lambda)} .
$$

Proof. Since $\mathcal{I}$ is a dissipative extension of the symmetric operator $L_{0}$, we have $G_{\mathfrak{J}}=M_{\mathfrak{J}} \cap M_{\mathcal{J}^{*}}$. Moreover, $\mathrm{F}(\mathcal{J})=\mathrm{M}_{\mathfrak{J}} / \mathrm{G}_{\mathfrak{J}}$ and $\mathrm{F}_{*}(\mathcal{J})=\mathrm{M}_{\mathcal{J}} / \mathrm{G}_{\mathfrak{J}}$.

Let $\mathcal{P}$ and $\mathcal{P}_{*}$ denote the natural projections such that $\mathcal{P}: M_{\mathcal{J}} \rightarrow F(\mathcal{J})$ and $\mathcal{P}_{*}: M_{\mathcal{J}^{*}} \rightarrow F_{*}(\mathcal{J})$. From (2.10) we have for $y=\left\{y_{n}\right\}_{-\infty}^{\infty} \in F(\mathcal{J})$ that

$$
\|\mathcal{P} y\|_{\mathrm{F}}^{2}=\operatorname{Imh}|[y, z](\infty)|^{2} .
$$

With a similar calculation for $\chi=\left\{\chi_{n}\right\}_{-\infty}^{\infty} \in F_{*}(\mathcal{J})$ that

$$
\left\|\mathcal{P}_{*} \chi\right\|_{\mathrm{F}_{*}}^{2}=\operatorname{Imh}|[\chi, z](\infty)|^{2} .
$$

We set $\mathrm{E}=\mathrm{E}_{*}=\mathbb{C}$ and we define the isometric isomorphisms $\Psi, \Psi_{*}$ :

$$
\begin{aligned}
\Psi: \quad E & \rightarrow F(\mathcal{J}), \\
& a \rightarrow \Psi(a)=\mathcal{P} y,
\end{aligned}
$$

$y \in M_{\mathfrak{\jmath}}$, and

$$
\begin{aligned}
\Psi_{*}: & E_{*} \rightarrow F_{*}(\mathcal{J}), \\
& a \rightarrow \Psi_{*}(a)=\mathcal{P}_{*} \chi,
\end{aligned}
$$

$\chi \in M_{\mathcal{J}^{*}}$, such that

$$
[y, z](\infty)=\frac{a}{\sqrt{\mathrm{Imh}}},[x, z](\infty)=\frac{\mathrm{a}}{\sqrt{\mathrm{Imh}}} .
$$

Taking $A=\mathcal{J}$ in (3.5) we get that the characteristic function of $\mathcal{J}$ is as the following form

$$
S_{\mathfrak{J}}(\lambda)=\Psi_{*}^{*} \mathcal{P}_{*}\left(\mathcal{J}^{*}-\lambda \mathbf{1}\right)^{-1}(\mathcal{J}-\lambda \mathbf{1}) \mathcal{P}^{-1} \Psi
$$


Using (3.6) we obtain from (3.8) that

$$
S_{\mathcal{J}}(\lambda) a=\Psi_{*}^{*} \mathcal{P}_{*} \chi
$$

where

$$
x=\left(\mathcal{J}^{*}-\lambda \mathbf{1}\right)^{-1}(\mathcal{J}-\lambda \mathbf{1}) y,
$$

$y \in M_{\mathcal{J}}$ with $[y, z](\infty)=a / \sqrt{\operatorname{Imh}}$. Finally, using (3.7) we obtain $\Psi_{*}^{*} \mathcal{P}_{* \chi}=[\chi, z](\infty) \sqrt{\operatorname{Imh}}$. In other words, we obtain from (3.9) that

$$
S_{\mathfrak{J}}(\lambda) a=\frac{[\chi, z](\infty)}{[y, z](\infty)} a .
$$

Equation (3.10) particularly implies that

$$
\left(\mathfrak{J}^{*}-\lambda \mathbf{1}\right) \chi=(\mathcal{J}-\lambda \mathbf{1}) y,
$$

where $\chi \in M_{\mathcal{J}^{*}}$ and $y \in M_{\mathcal{J}}$. Therefore $\chi-y$ can be represented as a solution of $J \psi=\lambda \psi$ with $\psi \in \ell^{2}(\mathbb{Z})$ satisfying $[\psi, u](-\infty)-l[\psi, z](-\infty)=0$. Consequently, $x-y$ is a multiple of the solution $P=P(\lambda)$ : $x-y=c P$. Since $[y, u](\infty)-h[y, z](\infty)=0$ and $[x, u](\infty)-\bar{h}[x, z](\infty)=0$ we obtain from the equation

$$
[\mathrm{P}(\lambda), \mathrm{u}](\infty)=\frac{[\mathrm{P}(\lambda), \mathrm{u}](\infty)}{[\mathrm{P}(\lambda), z](\infty)}[\mathrm{P}(\lambda), z](\infty)
$$

that

$$
\{[\mathrm{P}(\lambda), \mathrm{u}](\infty)-\overline{\mathrm{h}}[\mathrm{P}(\lambda), z](\infty)\}[\chi, z](\infty)=\{[\mathrm{P}(\lambda), \mathrm{u}](\infty)-\mathrm{h}[\mathrm{P}(\lambda), z](\infty)\}[y, z](\infty) .
$$

Substituting (3.12) in (3.11) we find

$$
S_{\mathcal{J}}(\lambda) a=\frac{[P(\lambda), u](\infty)-h[P(\lambda), z](\infty)}{[P(\lambda), u](\infty)-\bar{h}[P(\lambda), z](\infty)} a
$$

Therefore the proof is completed.

Since there is a connection between the characteristic functions of $\mathcal{J}$ and $\mathcal{T}$ with the rule

$$
S_{\mathcal{J}}(\lambda)=\Theta_{\mathcal{T}}\left(\frac{\lambda-i}{\lambda+i}\right), S_{\mathcal{J}}: E \rightarrow E_{*}, \operatorname{Im} \lambda>0,
$$

we obtain the following theorem.

Theorem 3.8. The characteristic function of $\mathcal{T}$ is

$$
\Theta_{\mathcal{T}}(\mu)=\frac{\omega_{h}(\lambda)}{\omega_{\bar{h}}(\lambda)}, \mu=\frac{\lambda-i}{\lambda+i}, \operatorname{Im} \lambda>0 .
$$

To investigate the spectral properties of the c.n.u. contraction $\mathcal{T}$, we shall obtain that $\mathcal{T}$ belongs to the class $C_{0}$. Therefore following lemma is useful. Note the the idea belongs to Nikolskir [19].

Lemma 3.9. $\mathfrak{d}_{\mathcal{T}}=\mathfrak{d}_{\mathcal{T}^{*}}=1$.

Proof. For $y=\left\{y_{n}\right\}_{-\infty}^{\infty} \in M_{\mathfrak{f}}$, let us consider the equation $(\mathfrak{d}+\mathrm{i} \mathbf{1}) \mathrm{y}=\mathrm{f}$, where $f=\left\{f_{n}\right\}_{-\infty}^{\infty}$. Following [19], we obtain

$$
D_{\mathcal{T}}^{2} f=(\mathcal{J}+i \mathbf{1}) y-\left(\mathcal{J}^{*}+i \mathbf{1}\right) \chi
$$

where

$$
x=\left(\mathcal{J}^{*}-\mathfrak{i 1}\right)^{-1}(\mathcal{J}-\mathbf{i} \mathbf{1}) y .
$$

Consequently, one gets

$$
\mathcal{J}(y-x)=i(y-x) .
$$


Note that $\chi=\left\{\chi_{n}\right\}_{-\infty}^{\infty} \in M_{\mathcal{J}^{*}}$. Therefore, $y-\chi$ is a multiple of $P=P(\lambda): y-\chi=c P$. Using this in (3.13) we obtain

$$
D_{\mathcal{T}}^{2} f=(\mathcal{J}+i \mathbf{1})(y-\chi)=2 i c P(i) .
$$

This implies that $\mathfrak{D}_{\mathcal{T}}$ is spanned by $P(i)$, i.e., $\mathfrak{d}_{\mathcal{T}}=1$.

Now let $y=\left\{y_{n}\right\}_{-\infty}^{\infty} \in M_{\mathcal{J}^{*}}$ and $\left(\mathcal{J}^{*}-i \mathbf{1}\right) y=f$, where $f=\left\{f_{n}\right\}_{-\infty}^{\infty}$. Then

$$
D_{\mathcal{T}^{*}}^{2} f=\left(\mathfrak{J}^{*}-i \mathbf{1}\right) y-(\mathcal{J}-i \mathbf{1}) \chi,
$$

where

$$
x=(\mathcal{J}+i \mathbf{1})^{-1}\left(\mathcal{J}^{*}+\mathfrak{i} \mathbf{1}\right) y .
$$

Therefore

$$
\mathcal{J}(y-x)=i(x-y)
$$

Hence (3.14) gives

$$
D_{\mathcal{T}^{*}}^{2} f=(\mathcal{J}-\mathbf{i} \mathbf{1})(y-\chi)=2 i d P(-\mathfrak{i}) .
$$

This implies that $\mathfrak{D}_{\mathcal{T}^{*}}$ is spanned by $\mathrm{P}(-\mathfrak{i})$, i.e., $\mathfrak{d}_{\mathcal{T}^{*}}=1$. The proof is completed.

The characteristic function of the adjoint operator $\mathcal{T}^{*}$ can be obtained with the aid of the characteristic function of $\mathcal{T}$. Indeed, the following equality is known

$$
\Theta_{\mathcal{T} *}(\mu)=\Theta_{\mathcal{T}}^{*}(\bar{\mu}), \mu \in \mathbf{D} .
$$

Therefore we have the following corollary.

Corollary 3.10. The characteristic function of $\mathcal{T}^{*}$ is

$$
\Theta_{\mathcal{T}^{*}}(\mu)=\frac{\omega_{\bar{h}}\left(-i \frac{1+\mu}{1-\mu}\right)}{\omega_{h}\left(-i \frac{1+\mu}{1-\mu}\right)}, \mu=\frac{\lambda-i}{\lambda+i}, \operatorname{Im} \lambda>0 .
$$

Using Theorem 3.5 and Lemma 3.9, we have the following theorem.

Theorem 3.11. The c.n.u. contraction $\mathcal{T}$ belongs to the class $\mathrm{C}_{0}$. Moreover, the characteristic function $\Theta_{\mathcal{T}}(\mu)$ of $\mathcal{T}$ coincides with the minimal function $\mathrm{m}_{\mathcal{T}}(\mu)$ of $\mathcal{T}$.

Remark 3.12. 1 is not an eigenvalue of $\mathcal{T}$. On the other side, the spectrum of $\mathcal{T}$ coincides with those $\mu$ belong to the disc $\mathbf{D}$ for which the operator $\Theta_{\mathcal{T}}(\mu)$ is not boundedly invertible, together with those $\mu \in \mathbf{C}$ not lying on any of the open arcs of $\mathbf{C}$ on which $\Theta_{\mathcal{T}}(\mu)$ is a unitary operator-valued analytic function of $\mu$ and point spectrum of $\mathcal{T}$ coincides with those $\mu \in D$ for which $\Theta_{\mathcal{T}}(\mu)$ is not invertible at all. Since the zeros of $\omega_{h}(\lambda), \operatorname{Im} \lambda>0$, are eigenvalues of $\mathcal{J}, \lambda=\mathfrak{i}(1+\mu) /(1-\mu)$ for $\lambda=i$ s, $\lim _{s \rightarrow \infty}(i s)=: \lambda_{\infty}$ can not be a zero of $\omega_{h}(\lambda)$ or equivalently an eigenvalue of $\mathcal{J}$.

Remark 3.13. Since $\mathcal{T} \in C_{.0}, \Theta_{\mathcal{T}}(\mu)$ is an inner function.

Theorem 3.14. $\Theta_{\mathcal{T}}(\mu)$ is a Blaschke product in the disc $\mu \in \mathbf{D}$ or equivalently in the open upper half-plane $\operatorname{Im} \lambda>0$.

Proof. Remarks 3.12 and 3.13 imply that $\Theta_{\mathcal{T}}(\lambda), \operatorname{Im} \lambda \geqslant 0$, has the representation

$$
\Theta_{\mathcal{T}}(\lambda)=B(\lambda) \exp (i \lambda b), b>0,
$$

where $B(\lambda)$ is a Blashke product. Therefore

$$
\left|\Theta_{\mathcal{T}}(\lambda)\right| \leqslant \exp (-\mathrm{b} \operatorname{Im} \lambda) .
$$

Letting $\lambda_{s}=$ is and $s \rightarrow \infty$ we obtain from (3.16) that $\omega_{h}\left(\lambda_{\infty}\right)=0$, that is, $\lambda_{\infty}=\lim _{s \rightarrow \infty}$ is is a zero of $\omega_{h}$ or equivalently is an eigenvalue of $\mathcal{J}$. However, as was pointed out in Remark 3.12 that this is not possible. Therefore there is not a singular factor in the factorization (3.15) and this completes the proof. 
According to the well-known theorem of Nagy and Foiaş [16] we obtain the following result.

Theorem 3.15. Root functions of $\mathcal{T}$ associated with the points of the spectrum of $\mathcal{T}$ in $\mathbf{D}$ span the Hilbert space $\ell^{2}(\mathbb{Z})$.

Definition 3.16. Let all root functions of the operator $A$ span the Hilbert space $H$. Such an operator is called complete operator. If every $A$-invariant subspace is generated by root vectors of $A$ belonging to the subspace then it is said $A$ admits spectral synthesis.

Since every complete operator in $\mathrm{C}_{0}$ admits spectral synthesis $[18,19]$, we obtain the following.

Theorem 3.17. $\mathcal{T}$ admits spectral synthesis.

Since the root functions of $\mathcal{T}$ span $\ell^{2}(\mathbb{Z})$ then those of $\mathcal{J}$ must span $\ell^{2}(\mathbb{Z})[7$, p. 42] . Therefore, we have the following.

Theorem 3.18. Root functions of $\mathcal{J}$ associated with the point spectrum of $\mathcal{J}$ in the open upper half-plane $\operatorname{Im} \lambda>0$ span the Hilbert space $\ell^{2}(\mathbb{Z})$.

Root functions of $\mathcal{\partial}$ and eigen and associated functions of $\mathcal{\partial}$ coincide. Moreover, the eigenvalue problem of $\mathcal{f}$ coincides with the eigenvalue problem of the problem (2.6). Therefore we have the following corollary.

\section{Corollary 3.19.}

(i) Eigenvalues of (2.6) are countable in the open upper half-plane;

(ii) infinity is the only possible limit point of the eigenvalues of (2.6);

(iii) infinity must belong to the spectrum of (2.6), however, may not be an eigenvalue of (2.6);

(iv) the system of all eigen and associated functions of the problem (2.6) span the Hilbert space $\ell^{2}(\mathbb{Z})$.

\section{Selfadjoint dilation}

\subsection{Selfadjoint dilation}

Let $A$ be a maximal dissipative operator in a Hilbert space $H$ and $\mathcal{L}$ be a self-adjoint operator in another Hilbert space $\mathcal{H}$. If one of the followings holds,

(i) $(\mathrm{A}-\lambda \mathrm{I})^{-1}=\mathrm{P}_{\mathrm{H}}(\mathcal{L}-\lambda \mathrm{I})^{-1} \mid \mathrm{H}, \lambda \in \mathbb{C}$;

(ii) $(A+i I)^{-n}=P_{H}(\mathcal{L}+i I)^{-n} \mid H, n \geqslant 0$;

(iii) $\exp ($ iAt $)=P_{H} \exp (i \mathcal{L} t) \mid H, t>0$;

(iv) the operator $(L-i I)(L+i I)^{-1}$ is a unitary dilation of $(A-i I)(A+i I)^{-1}$,

then $\mathcal{L}$ is called a self-adjoint dilation of $A$ [26].

If $A$ is a maximal dissipative operator in $H$ with finite deficiency indices and $G_{A}$ is dense in $H$, then the self-adjoint dilation of $A$ can be given as the following theorem [26].

Theorem 4.1. Let $\mathrm{A}$ be a maximal dissipative operator with domain $\mathrm{D}(\mathrm{A})$ and finite deficiency indices such that $\mathrm{G}_{\mathrm{A}}$ is dense in $\mathrm{H}$. Then its self-adjoint dilation has the form

$$
\mathcal{A}\left\langle v_{-}, \mathrm{f}, v_{+}\right\rangle=\left\langle i v_{-}^{\prime}, \widetilde{A} f, i v_{+}^{\prime}\right\rangle,
$$

where the domain $\mathrm{D}(\mathcal{A})$ consists of those vector-functions $\left\langle v_{-}, \mathrm{f}, v_{+}\right\rangle$such that

$$
v_{-} \in W_{2}^{1}\left(\mathbb{R}_{-}, E_{*}\right), v_{+} \in W_{2}^{1}\left(\mathbb{R}_{+}, E_{*}\right)
$$

and

$$
f-\frac{i}{\sqrt{2}}\left(\Psi_{*} v_{-}(0)\right) \in D(A), f+\frac{i}{\sqrt{2}}\left(\Psi v_{+}(0)\right) \in D\left(A^{*}\right) .
$$

Here $\mathrm{E}$ and $\mathrm{E}_{*}$ are auxiliary Hilbert spaces which are isomorphic to $\mathfrak{D}_{\mathcal{T}}$ and $\mathfrak{D}_{\mathcal{T}^{*}}$, respectively, $\mathbb{R}_{-}=(-\infty, 0]$, $\mathbb{R}_{+}=[0, \infty)$, and

$$
\widetilde{A} f:=\left(A \mid G_{A}\right)^{*}= \begin{cases}A, & \text { on } D(A) \\ A^{*}, & \text { on } D\left(A^{*}\right)\end{cases}
$$


Then we have the following theorem.

Theorem 4.2. The self-adjoint dilation $\mathbf{J}$ of the operator $\mathcal{J}$ acts on the direct sum Hilbert space $\mathrm{L}^{2}\left(\mathbb{R}_{-}\right) \oplus \ell^{2}(\mathbb{Z}) \oplus$ $\mathrm{L}^{2}\left(\mathbb{R}_{+}\right)$and has the following form

$$
\mathrm{J}\left\langle\mathrm{v}_{-}, \mathrm{f}, \mathrm{v}_{+}\right\rangle=\left\langle i v_{-}^{\prime}, \mathrm{Jf}, \mathfrak{i} v_{+}^{\prime}\right\rangle,
$$

where $v_{-} \in \mathrm{W}_{2}^{1}\left(\mathbb{R}_{-}\right), v_{+} \in \mathrm{W}_{2}^{1}\left(\mathbb{R}_{+}\right)$such that

$$
[f, u](\infty)-h[f, z](\infty)=\sqrt{2 \operatorname{Imh}} v_{-}(0),[f, u](\infty)-\bar{h}[f, z](\infty)=\sqrt{2 \operatorname{Imh}} v_{+}(0) .
$$

Proof. Let us consider the sums $\eta_{-}:=f-\frac{i}{2}\left(\Psi_{*} v_{-}(0)\right) \in M_{\mathcal{J}}$ and $\eta_{+}:=f+\frac{i}{2}\left(\Psi v_{+}(0)\right) \in M_{\mathcal{J}^{*}}$. Let $y=\left\{y_{n}\right\}_{-\infty}^{\infty} \in M_{\mathcal{J}}$ with $[y, z](\infty)=v_{+}(0) / \sqrt{\operatorname{Imh}}$ and $\chi=\left\{\chi_{n}\right\}_{-\infty}^{\infty} \in M_{\mathcal{J} *}$ with $\left[\chi_{,} z\right](\infty)=v_{-}(0) / \sqrt{\operatorname{Imh}}$. Since $\eta_{-} \in M_{\mathcal{J}}$ one has

$$
[\mathrm{f}, \mathrm{u}](\infty)-\frac{i}{\sqrt{2}}[\chi, u](\infty)=h[f, z](\infty)-\frac{i}{\sqrt{2}} h[\chi, z](\infty)
$$

and hence

$$
[\mathrm{f}, \mathrm{u}](\infty)-\mathrm{h}[\mathrm{f}, z](\infty)=\sqrt{2 \operatorname{Imh}} v_{-}(0) .
$$

In a similar manner, $\eta_{+} \in M_{\mathcal{J} *}$ implies

$$
[f, u](\infty)+\frac{i}{\sqrt{2}}[y, u](\infty)=\bar{h}[f, z](\infty)+\frac{i}{\sqrt{2}} \bar{h}[y, z](\infty)
$$

and consequently we get

$$
[f, u](\infty)-\bar{h}[f, z](\infty)=\sqrt{2 \operatorname{Imh}} v_{+}(0) .
$$

Therefore the proof is completed.

\subsection{Functional embeddings}

Let $A$ be a maximal dissipative operator in a Hilbert space $H$ and $\mathcal{A}$ be its minimal self-adjoint dilation in the space

$$
\mathcal{H}=\mathrm{G}_{*} \oplus \mathrm{H} \oplus \mathrm{G},
$$

where $\mathrm{G}$ is the incoming subspace and $\mathrm{G}_{*}$ is the outgoing subspace such that

$$
\exp (\mathrm{i} \mathcal{A} \mathrm{t}) \mathrm{G} \subset \mathrm{G}, \mathrm{t}>0 ; \exp (\mathrm{i} \mathcal{A} \mathrm{t}) \mathrm{G}_{*} \subset \mathrm{G}_{*}, \mathrm{t}<0 .
$$

Consider the following isometries

$$
\pi^{\mathbb{R}}: L^{2}(\mathbb{R}, E) \rightarrow H, \operatorname{dim} E=\operatorname{dim} F(A),
$$

and

$$
\pi_{*}^{\mathbb{R}}: \mathrm{L}^{2}\left(\mathbb{R}, \mathrm{E}_{*}\right) \rightarrow \mathrm{H}, \operatorname{dim} \mathrm{E}_{*}=\operatorname{dim} \mathrm{F}_{*}(\mathrm{~A}),
$$

such that $(\mathcal{A}+i \mathrm{I})^{-1} \pi^{\mathbb{R}}=\pi^{\mathbb{R}}(Z+i \mathrm{I})^{-1},(\mathcal{A}+i \mathrm{I})^{-1} \pi_{*}^{\mathbb{R}}=\pi_{*}^{\mathbb{R}}(Z+i \mathrm{I})^{-1}, \pi^{\mathbb{R}} \mathrm{H}^{2}\left(\mathbb{C}_{+}, \mathrm{E}\right)=\mathrm{G}, \pi_{*}^{\mathbb{R}} \mathrm{H}^{2}\left(\mathbb{C}_{-}, \mathrm{E}_{*}\right)=$ $\mathrm{G}_{*}$, and $\mathcal{A} \mid \mathrm{G}=\mathrm{Z}$. $\pi^{\mathbb{R}}$ and $\pi_{*}^{\mathbb{R}}$ are called functional embeddings. If $\mathrm{T}=(\mathrm{A}-\mathrm{iI})(\mathrm{A}+\mathrm{iI})^{-1}$, then

$$
\pi^{\mathbb{R}}: \pi \circ W^{-1}, \pi_{*}^{\mathbb{R}}: \pi_{*} \circ W^{-1},
$$

where $\pi, \pi_{*}$ are functional models of $\mathrm{T}$,

$$
(W f)(x)=\frac{1}{\sqrt{\pi}} \frac{1}{x+i} f\left(\frac{x-i}{x+i}\right)
$$

and $W$ is an isometric isomorphism of $L^{2}(D, \mathcal{E})$ onto $L^{2}(\mathbb{R}, \mathcal{E})$ for any space $\mathcal{E}$. 
The operator $S=\left(\pi_{*}^{\mathbb{R}}\right)^{*} \pi^{\mathbb{R}}$ acts from $L^{2}(\mathbb{R}, E)$ into $L^{2}\left(\mathbb{R}, E_{*}\right)$, maps $H^{2}\left(\mathbb{C}_{+}, E\right)$ into $H^{2}\left(\mathbb{C}_{+}, E_{*}\right)$, and commutes with multiplication $(z+i)^{-1}$. Consequently, $S$ is a multiplication by a function $S_{A} \in H^{\infty}$, $S_{A}: E \rightarrow E_{*} . S_{A}$ is called the characteristic function of $A$. Moreover

$$
S_{A}(\lambda)=\Theta_{T}\left(\frac{\lambda-i}{\lambda+i}\right)
$$

where $\Theta_{\mathrm{T}}$ is the characteristic function of $\mathrm{T}$.

Hence generalized eigenfunctions of the dilation can be described by the characteristic function of the maximal dissipative operator $A$. In fact, incoming eigenfunctions are of the form

$$
\left\langle S(\lambda) \exp (-i \lambda \xi) d, \frac{i}{\sqrt{2}}\left(\left(A^{*}-\bar{\lambda} I\right)^{-1}(A-\bar{\lambda} I)-I\right) \mathcal{P}^{-1} \Psi d, \exp (-i \lambda \zeta) d\right\rangle,
$$

and outgoing eigenfunctions are of the form

$$
\left\langle\exp (-i \lambda \xi) e,-\frac{i}{\sqrt{2}}\left((A-\lambda I)^{-1}\left(A^{*}-\lambda I\right)-I\right) \mathcal{P}_{*}^{-1} \Psi_{*} e, S^{*}(\lambda) \exp (-i \lambda \zeta) e\right\rangle,
$$

where $\xi \in \mathbb{R}_{-}, \zeta \in \mathbb{R}_{+}, \mathrm{d} \in \mathrm{E}, e \in \mathrm{E}_{*}$, and $\lambda \in \mathbb{R}$.

Hence we have the following theorem.

Theorem 4.3. The incoming eigenfunction of the dilation $\mathrm{J}$ is

$$
\left\langle\frac{\omega_{h}(\lambda)}{\omega_{\bar{h}}(\lambda)} \exp (-i \lambda \xi), \sqrt{2 \operatorname{Imh}}([\mathrm{P}(\bar{\lambda}), u](\infty)-h[P(\bar{\lambda}), z](\infty))^{-1} \mathrm{P}(\bar{\lambda}), \exp (-i \lambda \zeta)\right\rangle
$$

and outgoing eigenfunction of $\mathrm{J}$ is

$$
\left\langle\exp (-\mathfrak{i} \lambda \xi), \sqrt{2 \operatorname{Imh}}([\mathrm{P}(\lambda), u](\infty)-\overline{\mathrm{h}}[\mathrm{P}(\lambda), z](\infty))^{-1} \mathrm{P}(\lambda), \frac{\omega_{\bar{h}}(\bar{\lambda})}{\omega_{\mathrm{h}}(\bar{\lambda})} \exp (-\mathfrak{i} \lambda \zeta)\right\rangle,
$$

where $\xi \in \mathbb{R}_{-}, \zeta \in \mathbb{R}_{+}$, and $\lambda \in \mathbb{R}$.

Proof. Consider the equation

$$
\left(\left(\mathcal{J}^{*}-\bar{\lambda} \mathbf{1}\right)^{-1}(\mathcal{J}-\bar{\lambda} \mathbf{1})-\mathbf{1}\right) \mathcal{P}^{-1} \Psi \mathrm{a}=\mathrm{cP}(\bar{\lambda}),
$$

where $x-y=\operatorname{cP}(\lambda), x \in M_{\mathfrak{\jmath} *}$, and $y \in M_{\mathfrak{\jmath}}$. Then we have

$$
c[\mathrm{P}(\bar{\lambda}), z](\infty)=[\bar{\chi}, z](\infty)-[\bar{y}, z](\infty)=\frac{2 i \operatorname{Imh}[\mathrm{P}(\bar{\lambda}), z](\infty)}{[\mathrm{P}(\bar{\lambda}), \mathrm{u}](\infty)-\mathrm{h}[\mathrm{P}(\bar{\lambda}), z](\infty)} \frac{\mathrm{d}}{\sqrt{\operatorname{Imh}}}
$$

Therefore we get

$$
c=c(\lambda)=\frac{2 i \sqrt{\operatorname{Imh}}}{[P(\bar{\lambda}), u](\infty)-h[P(\bar{\lambda}), z](\infty)} d .
$$

Substituting (4.5) into (4.1) we obtain (4.3).

Similarly consider the equation

$$
\left((\mathcal{J}-\lambda \mathbf{1})^{-1}\left(\mathcal{J}^{*}-\lambda \mathbf{1}\right)-\mathbf{1}\right) \mathcal{P}_{*}^{-1} \Psi_{*} e=c P(\lambda),
$$

where $y-\chi=c P(\lambda), \chi \in M_{\mathcal{J}^{*}}$, and $y \in M_{\mathcal{J}}$. Hence we have

$$
c=c(\lambda)=\frac{2 i \sqrt{\operatorname{Imh}}}{[P(\lambda), u](\infty)-\bar{h}[P(\lambda), z](\infty)} d .
$$

Substituting (4.6) into (4.2) we obtain (4.4). The proof is completed. 


\section{Other properties of $\mathcal{T}$}

\subsection{Multiplicity of $\mathcal{T}$}

Let us consider the linear mappings $\mathrm{A}: \mathrm{X} \rightarrow \mathrm{X}, \mathrm{B}: \mathrm{U} \rightarrow \mathrm{X}, \mathrm{C}=\mathrm{X} \rightarrow \mathrm{Y}$ and $\mathrm{D}: \mathrm{U} \rightarrow \mathrm{Y}$, where $\mathrm{X}, \mathrm{Y}$ and $\mathrm{U}$ are Banach spaces and following linear dynamic system $[19,20]$

$$
\begin{aligned}
x^{\prime}(t) & =A x(t)+B u(t), t \geqslant 0, \\
y(t) & =C x(t)+D u(t), t \geqslant 0,
\end{aligned}
$$

with $x(0)=x_{0}, t \geqslant 0$. Here $x(t) \in X$ and $u(t) \in U$. The operators $A, B$ are called the generator operator and control operator, respectively, while $\mathrm{C}$ and $\mathrm{D}$ are called observation operators. $\mathrm{X}$ is called state space and $x(\mathrm{t})$ is the state of the system at time $t$. Finally, $u$ is the input function, $y$ is the output function, and $x_{0}$ is the initial state.

It is meant by controlling the system (5.1)-(5.2) to act on the system with the help of the function $u$ in such a way that starting from a given initial state $x_{0}$ the system attains a time $\tau>0$ the final state $x_{1}=x(\tau), \tau>0$. The system (5.1) is called approximately controllable if for every $x_{0}, x_{1} \in X$ and arbitrary $\epsilon>0$ there exist $\tau \in[0, \infty)$ and $u \in \mathrm{L}^{2}(0, \tau)$ such that

$$
\left\|x(\tau)-x_{1}\right\|<\epsilon, \quad x(0)=x_{0} .
$$

Let the system (5.1) be controllable. This is referred as the system $(A, B)$. Let $S(\cdot)$ be a semigroup associated with $A$. The system $(A, B)$ is approximately controllable on $[0, \infty)$ if and only if

$$
\operatorname{span}\{S(t) B U: t \geqslant 0\}=X .
$$

If the generator $A$ is bounded, then (5.3) is satisfied if and only if

$$
\operatorname{span}\left\{A^{n} B U: n \geqslant 0\right\}=X .
$$

Therefore, it is important to find the least possible dimension of the control subspace dim BU such that $(A, B)$ is approximately controllable, i.e.,

$$
\min \{\operatorname{dim} B U:(A, B) \text { is approximately controllable }\} \text {. }
$$

For an arbitrary bounded operator $\mathrm{T}: \mathrm{X} \rightarrow \mathrm{X}$ the multiplicity of the spectrum is defined by

$$
\boldsymbol{\mu}_{\mathrm{T}}=\min \left\{\operatorname{dim} C: \operatorname{span}\left(\mathrm{T}^{\mathrm{n}} \mathrm{C}: \mathrm{n} \geqslant 0\right)=X\right\} .
$$

$\mathrm{T}$ is called multiplicity-free if $\boldsymbol{\mu}_{\mathrm{T}}=1$.

One can infer that $T$ is unitary equivalent to the model operator $Z: \mathrm{H}_{\Theta} \rightarrow \mathrm{H}_{\Theta}$, where

$$
\mathrm{H}_{\Theta}=\left(\mathrm{H}^{2} \oplus \operatorname{clos} \Lambda \mathrm{L}^{2}\right) \ominus(\Theta \oplus \Lambda) \mathrm{H}^{2},
$$

$L^{2}=L^{2}(\mathbf{C}), H^{2}$ is the Hardy space, $\Lambda=\left(1-|\Theta|^{2}\right)^{1 / 2}, Z f=P_{\Theta} z f, f \in H_{\Theta}, P_{\Theta}=\Theta_{-} \bar{\Theta}_{\text {, }}$ and $P_{-}$is the projection of $\mathrm{L}^{2}$ into $\mathrm{H}_{-}^{2}$ (the Hardy space in the lower half plane).

A c.n.u. contraction $T$ with a scalar characteristic function may help one to compute the multiplicity of T. This method was constructed in [19]. Since $\Theta \neq 0$, we obtain the following theorem ([19, p. 247]).

Theorem 5.1. $\boldsymbol{\mu}_{\mathcal{T}}=1$.

In general, the adjoint of a multiplicity-free operator is not generally multiplicity-free. However, since $\mathcal{T} \in \mathrm{C}_{0}$ we can find the multiplicity of $\mathcal{T}^{*}$. Before this, we shall give some definitions. 
Definition 5.2. Let $V$ be an isometry on the Hilbert space $H$. A subspace $\mathcal{L}$ of $H$ is called a wandering space for $V$ if $V^{p} \mathcal{L} \perp V^{q} \mathcal{L}$ for every pair of integers $p, q \geqslant 0, p \neq q$. An isometry $V$ on $H$ is called a unilateral shift if there exists in $\mathrm{H}$ a subspace $\mathcal{L}$ which is wandering for $\mathrm{V}$ and such that

$$
\mathrm{H}=\bigoplus_{0}^{\infty} \mathrm{v}^{\mathrm{n}} \mathcal{L} .
$$

The dimension of $\mathrm{H} \ominus \mathrm{V} \mathcal{L}$ is called the multiplicity of the unilateral shift $\mathrm{V}$.

Let $\mathrm{S}$ denote the unilateral shift of multiplicity one acting on $\mathrm{H}^{2}$.

Definition 5.3. For each inner function $\varphi \in H^{\infty}$, the Jordan block $S(\varphi)$ is the operator defined on $H(\varphi)=$ $\mathrm{H}^{2} \ominus \varphi \mathrm{H}^{2}$ by $\mathrm{S}(\varphi)=\mathrm{P}_{\mathrm{H}(\varphi)} \mathrm{S} \mid \mathrm{H}(\varphi)$ or equivalently, $\mathrm{S}(\varphi)^{*}=\mathrm{S}^{*} \mid \mathrm{H}(\varphi)$.

Definition 5.4. By an affinity from $\mathrm{H}_{1}$ to $\mathrm{H}_{2}$ it is meant a linear, one-to-one, and bicontinuous transformation $X$ from $\mathrm{H}_{1}$ onto $\mathrm{H}_{2}$. Thus bounded operators, say $\mathrm{S}_{1}$ on $\mathrm{H}_{1}$ and $\mathrm{S}_{2}$ on $\mathrm{H}_{2}$, are said to be similar if there exists an affinity $X$ from $H_{1}$ to $H_{2}$ such that $X S_{1}=S_{2} X$ (and consequently $X^{-1} S_{2}=S_{1} X$ ).

Definition 5.5. By a quasi-affinity from $\mathrm{H}_{1}$ to $\mathrm{H}_{2}$ it is meant a linear, one-to-one, and continuous transformation $X$ from $\mathrm{H}_{1}$ onto a dense linear manifold in $\mathrm{H}_{2}$ if $S_{1}$ and $S_{2}$ are bounded operators, $S_{1}$ on $H_{1}$ and $S_{2}$ on $H_{2}$, it is said that $S_{1}$ is a quasi-affine transform of $S_{2}$ if there exists a quasi-affinity $X$ from $H_{1}$ to $H_{2}$ such that $X S_{1}=S_{2} X$. The operators $S_{1}$ and $S_{2}$ are called quasi-similar if they are quasi-affine transforms of one another.

Definition 5.6. Let $L$ be a bounded operator in $H$, and let $\mathcal{L}$ be a subspace of $H$. $\mathcal{L}$ is said to be hyperinvariant for $L$ if it is invariant for every bounded operator which commutes with $L$.

Then we may introduce the following results [17, Chap. X, Sect. 4] .

\section{Theorem 5.7.}

(i) $\mathcal{T}^{*}$ is multiplicity-free, i.e., $\boldsymbol{\mu}_{\mathcal{T}^{*}}=1$;

(ii) $\mathcal{T}$ is quasi-similar to the Jordan block $\mathrm{S}\left(\frac{\omega_{\mathrm{h}}}{\omega_{\bar{h}}}\right)$;

(iii) $\mathcal{T} \mid \mathcal{C}$ is multiplicity-free, i.e., $\boldsymbol{\mu}_{\mathcal{T} \mid \mathcal{C}}=1$, where $\mathcal{C}$ is a invariant subspace of $\mathcal{T}$;

(iv) $\mathcal{C}$ is hyperinvariant, where $\mathcal{C}$ is a invariant subspace of $\mathcal{T}$.

\subsection{Unitary colligation}

Unitary colligation theory is more general than Sz.-Nagy-Foiaş characteristic function theory [8] and in recent years this theory has been developed by many authors (see [4] and references therein). Now we shall give some information about the unitary colligation theory. A set $\Delta=(\mathfrak{H}, \mathfrak{F}, \mathfrak{S} ; \mathrm{T}, \mathrm{F}, \mathrm{G}, \mathrm{S})$, where $\mathfrak{H}, \mathfrak{F}, \mathfrak{S}$ are seperable Hilbert spaces, is called a unitary colligation if the following block form

$$
\mathrm{U}=\left(\begin{array}{ll}
\mathrm{T} & \mathrm{F} \\
\mathrm{G} & \mathrm{S}
\end{array}\right)
$$

is a unitary mapping such that

$$
\mathrm{U}=\left(\begin{array}{cc}
\mathrm{T} & \mathrm{F} \\
\mathrm{G} & \mathrm{S}
\end{array}\right): \mathfrak{H} \oplus \mathfrak{F} \rightarrow \mathfrak{H} \oplus \mathfrak{S} .
$$

The spaces $\mathfrak{H}, \mathfrak{S}$, and $\mathfrak{F}$ are called, respectively, the inner, left-outer, and right-outer spaces and $\mathbf{U}$ is called the connecting operator. By $\mathrm{P}_{1}$ and $\mathrm{P}_{2}$ we denote the orthogonal projections of $\mathfrak{H} \oplus \mathfrak{S}$ onto $\mathfrak{H}$ and $\mathfrak{S}$, respectively. The operators

$$
\mathrm{T}=\mathrm{P}_{1}[\mathbf{U} \mid \mathfrak{H}], \mathrm{F}=\mathrm{P}_{1}[\mathbf{U} \mid \mathfrak{F}], \mathrm{G}=\mathrm{P}_{2}[\mathbf{U} \mid \mathfrak{H}], \mathrm{S}=\mathrm{P}_{2}[\mathbf{U} \mid \mathfrak{F}]
$$


are called the components of $\Delta$. The components T, F, G, and $S$ are called the basic, right-channeled, leftchanneled, and duplicating operators, respectively and following relations hold:

$$
\begin{aligned}
& \mathrm{T}^{*} \mathrm{~T}+\mathrm{G}^{*} \mathrm{G}=\mathrm{I}_{\mathfrak{H},}, \quad \mathrm{F}^{*} \mathrm{~F}+\mathrm{S}^{*} \mathrm{~S}=\mathrm{I}_{\mathfrak{F}}, \quad \mathrm{T}^{*} \mathrm{~F}+\mathrm{G}^{*} \mathrm{~S}=0, \\
& \mathrm{~T}^{*} \mathrm{~T}+\mathrm{F}^{*} \mathrm{~F}=\mathrm{I}_{\mathfrak{H}}, \quad \mathrm{GG}^{*}+\mathrm{SS}^{*}=\mathrm{I}_{\mathfrak{S},}, \quad \mathrm{TG}^{*}+\mathrm{FS}^{*}=0 .
\end{aligned}
$$

If one takes $\mathrm{F}=\mathrm{D}_{\mathrm{T}^{*}}, \mathrm{G}=\mathrm{D}_{\mathrm{T}}, \mathrm{S}=-\mathrm{T}^{*}, \mathfrak{F}=\mathfrak{D}_{\mathrm{T}^{*}}, \mathfrak{S}=\mathfrak{D}_{\mathrm{T}}$ then $\mathrm{U}$ also provides a unitary colligation.

The connecting operator $\mathbf{U}$ given in (5.4) can be given with a slightly different form:

$$
\mathrm{U}=\left(\begin{array}{cc}
\mathrm{S} & \mathrm{G} \\
\mathrm{F} & \mathrm{T}
\end{array}\right): \mathfrak{F} \oplus \mathfrak{H} \rightarrow \mathfrak{S} \oplus \mathfrak{H} .
$$

Consequently following block form gives a unitary colligation:

$$
\mathbf{U}=\left(\begin{array}{cc}
-\mathrm{T}^{*} & \mathrm{D}_{\mathrm{T}} \\
\mathrm{D}_{\mathrm{T}^{*}} & \mathrm{~T}
\end{array}\right): \mathfrak{D}_{\mathrm{T}^{*}} \oplus \mathrm{H} \rightarrow \mathfrak{D}_{\mathrm{T}} \oplus \mathrm{H}
$$

Let $\mathfrak{H}^{(\mathrm{c})}$ and $\mathfrak{H}^{(0)}$ be the subspaces of $\mathfrak{H}$ as follows [5]

$$
\mathfrak{H}^{(\mathrm{c})}=\overline{\operatorname{span}}\left\{\mathrm{T}^{\mathrm{n}} \mathrm{FF}, \mathrm{n}=0,1, \ldots\right\}, \quad \mathfrak{H}^{(\mathrm{o})}=\overline{\operatorname{span}}\left\{\mathrm{T}^{* \mathrm{n}} \mathrm{G}^{*} \mathfrak{S}, \mathrm{n}=0,1, \ldots\right\} .
$$

The subspaces $\mathfrak{H}^{(\mathfrak{c})}$ and $\mathfrak{H}^{(0)}$ are called the controllable and the observable subspaces, respectively. Let

$$
\left(\mathfrak{H}^{(\mathrm{c})}\right)^{\perp}:=\mathfrak{H} \ominus \mathfrak{H}^{(\mathrm{c})}, \quad\left(\mathfrak{H}^{(\mathrm{o})}\right)^{\perp}:=\mathfrak{H} \ominus \mathfrak{H}^{(\mathrm{o})} .
$$

A unitary colligation is called prime if $\overline{\mathfrak{H}^{(\mathrm{c})}+\mathfrak{H}^{(\mathrm{o})}}=\mathfrak{H}$. A unitary colligation $\Delta=(\mathfrak{F}, \mathfrak{S}, \mathfrak{H} ; S, G, F, T)$ associated with (5.5) is prime if and only if T is c.n.u. contraction. The characteristic function $\Theta_{\Delta}(\zeta)$ is defined by

$$
\Theta_{\Delta}(\zeta)=S+\zeta G\left(I_{\mathfrak{H}}-\zeta T\right)^{-1} \mathrm{~F}, \zeta \in \mathbf{D} .
$$

Following theorem describes all unitary colligations with basic operator $\mathrm{T}$.

Theorem 5.8 ([5, p. 163]). Let $\mathrm{T}$ be a contraction with $\mathfrak{d}_{\mathrm{T}}, \mathfrak{d}_{\mathrm{T}^{*}}<\infty$ acting on Hilbert space $\mathrm{H}$. Suppose that $\mathfrak{M}$ and $\mathfrak{N}$ are two Hilbert spaces such that $\operatorname{dim} \mathfrak{N}=\mathfrak{d}_{\mathrm{T}}$ and $\operatorname{dim} \mathfrak{M}=\mathfrak{d}_{T^{*}}$. Then all unitary colligations with the basic operator $\mathrm{T}$ and left-outer and right-outer subspaces $\mathfrak{M}$ and $\mathfrak{N}$ take the form $\Delta=\left(\mathfrak{M}, \mathfrak{N}, \mathrm{H} ;-\mathrm{KT}^{*} \mathrm{M}, \mathrm{KD}_{\mathrm{T}}, \mathrm{D}_{\mathrm{T}^{*}} \mathrm{M}, \mathrm{T}\right)$ such that

$$
\left(\begin{array}{cc}
-\mathrm{KT}^{*} \mathrm{M} & \mathrm{KD}_{\mathrm{T}} \\
\mathrm{D}_{\mathrm{T} *} \mathrm{M} & \mathrm{T}
\end{array}\right): \mathfrak{M} \oplus \mathrm{H} \rightarrow \mathfrak{N} \oplus \mathrm{H}
$$

where $\mathrm{K}: \mathfrak{D}_{\mathrm{T}} \rightarrow \mathfrak{N}$ and $\mathrm{M}: \mathfrak{M} \rightarrow \mathfrak{D}_{\mathrm{T} *}$ are unitary operators. The characteristic function of $\Delta$ is

$$
\Theta_{\Delta}(\zeta)=K \Theta_{\mathrm{T}^{*}}(\zeta) \mathrm{M}, \zeta \in \mathbf{D} .
$$

Now consider the unitary colligation $\Delta_{0}=\left(\mathfrak{D}_{\mathcal{T}^{*}}, \mathfrak{D}_{\mathcal{T}}, \ell^{2}(\mathbb{Z}) ;-\mathcal{T}^{*}, \mathrm{D}_{\mathcal{T}}, \mathrm{D}_{\mathcal{T}^{*}}, \mathfrak{T}\right)$. The characteristic function of $\Delta_{0}$ is

$$
\Theta_{\Delta_{0}}(\zeta)=\left[-\mathcal{T}^{*}+\zeta D_{\mathcal{T}}(\mathbf{1}-\zeta \mathcal{T})^{-1} D_{\mathcal{T}^{*}}\right] \mid \mathfrak{D}_{\mathcal{T}^{*}}
$$

Clearly $\Theta_{\Delta_{0}}(\zeta)$ is the characteristic function of $\mathcal{T}^{*}$. Hence we have

$$
\Theta_{\Delta_{0}}(\mu)=\frac{\omega_{\bar{h}}\left(-i \frac{1+\mu}{1-\mu}\right)}{\omega_{h}\left(-i \frac{1+\mu}{1-\mu}\right)}, \mu=\frac{\lambda-i}{\lambda+i}, \operatorname{Im} \lambda>0 .
$$

Because $\mathfrak{d}_{\mathcal{T}}=\mathfrak{d}_{\mathcal{T}^{*}}=1$, one may set the following isometric operators $\mathrm{K}: \mathfrak{D}_{\mathcal{T}} \rightarrow \mathbb{C}$ and $M: \mathbb{C} \rightarrow \mathfrak{D}_{\mathcal{T}^{*}}$. Now we consider the controllable and observable subspaces in $\mathrm{H}$ as follows

$$
\mathrm{H}^{(\mathrm{c})}=\overline{\operatorname{span}}\left\{\mathcal{T}^{\mathrm{n}} \mathrm{D}_{\mathcal{T}^{*}} M \mathbb{C}, \mathrm{n}=0,1, \ldots\right\}, \quad \mathrm{H}^{(\mathrm{o})}=\overline{\operatorname{span}}\left\{\mathcal{T}^{* n}\left(K \mathrm{D}_{\mathcal{T}}\right)^{*} \mathbb{C}, \mathrm{n}=0,1, \ldots\right\} .
$$

Let $\left(\mathrm{H}^{(\mathrm{c})}\right)^{\perp}=\ell^{2}(\mathbb{Z}) \ominus \mathrm{H}^{(\mathrm{c})},\left(\mathrm{H}^{(\mathrm{o})}\right)^{\perp}=\ell^{2}(\mathbb{Z}) \ominus \mathrm{H}^{(\mathrm{o})}$. Then using the results of [5] we give the following. 
Theorem 5.9. $\mathcal{T}=(\mathcal{J}-i \mathbf{1})(\mathcal{J}+i \mathbf{1})^{-1}$ can be included into the unitary colligation $\Delta_{0}=\left(\mathbb{C}, \mathbb{C}, \ell^{2}(\mathbb{Z}) ;-K \mathcal{T}^{*} M\right.$, $\left.\mathrm{KD}_{\mathcal{T}}, \mathrm{D}_{\mathcal{T} *} M, \mathcal{T}\right)$ as

$$
\mathbf{u}_{0}=\left(\begin{array}{cc}
-\mathrm{KT}^{*} M & \mathrm{KD}_{\mathcal{T}} \\
\mathrm{D}_{\mathcal{T}^{*} M} M & \mathcal{T}
\end{array}\right): \mathbb{C} \oplus \ell^{2}(\mathbb{Z}) \rightarrow \mathbb{C} \oplus \ell^{2}(\mathbb{Z})
$$

Let $\overrightarrow{1}=\left(\begin{array}{l}1 \\ 0\end{array}\right) \in \mathbb{C} \oplus \ell^{2}(\mathbb{Z})$. Then $\left(\mathrm{H}^{(\mathrm{c})}\right)^{\perp}=\left(\mathbb{C} \oplus \ell^{2}(\mathbb{Z})\right) \ominus \overline{\operatorname{span}}\left\{\mathbf{u}_{0}^{\mathrm{n}} \overrightarrow{1}, \mathrm{n}=0,1, \ldots\right\},\left(\mathrm{H}^{(\mathrm{o})}\right)^{\perp}=(\mathbb{C} \oplus$ $\left.\ell^{2}(\mathbb{Z})\right) \ominus \overline{\operatorname{span}}\left\{\mathbf{u}_{0}^{* n} \overrightarrow{1}, \mathrm{n}=0,1, \ldots\right\}$, and

(i) $\Delta_{0}$ is prime;

(ii) $\overrightarrow{1}$ is the cyclic vector for $\mathbf{u}_{0}: \overline{\operatorname{span}}\left\{\mathbf{u}_{0}^{n} \overrightarrow{1}, n \in \mathbb{Z}\right\}=\mathbb{C} \oplus \ell^{2}(\mathbb{Z})$.

All other unitary colligations with basic operator $\mathcal{T}$ and left- and right-outer spaces $\mathbb{C}$ are of the form $\widetilde{\Delta}_{0}=$ $\left(\mathbb{C}, \mathbb{C}, \ell^{2}(\mathbb{Z}) ;-\mathrm{d}_{1} \mathrm{~d}_{2} \mathcal{T}^{*}, \mathrm{~d}_{1} \mathrm{D}_{\mathcal{T}}, \mathrm{d}_{2} \mathrm{D}_{\mathcal{T}^{*}}, \mathcal{T}\right)$ with

$$
\widetilde{\mathbf{U}}_{0}=\left(\begin{array}{cc}
-\mathrm{d}_{1} \mathrm{~d}_{2} \mathrm{K \mathcal {T } ^ { * }} M & \mathrm{~d}_{1} \mathrm{KD}_{\mathcal{T}} \\
\mathrm{d}_{2} \mathrm{D}_{\mathcal{T}^{*} M} M & \mathcal{T}
\end{array}\right): \mathbb{C} \oplus \ell^{2}(\mathbb{Z}) \rightarrow \mathbb{C} \oplus \ell^{2}(\mathbb{Z})
$$

where $\left|\mathrm{d}_{1}\right|=\left|\mathrm{d}_{2}\right|=1$.

In the unit disc $D$, if a holomorphic function $F$ has the properties $\operatorname{Re} F>0$ and $F(0)=1$, then $F$ is called the Carathéodory function. For example,

$$
(F(\mathbf{U}) e, e)=\int_{\mathbf{C}} F(\zeta) \mathrm{dm}(\zeta)
$$

is a Carathéodory function, where $\mathbf{U}$ is a unitary operator with a cyclic vector acting on a Hilbert space and $\mathbf{m}$ is a nontrivial probability measure on the unit circle $\mathbf{C}$ (that is, not supported on a finite set) [5].

Since $\mathfrak{d}_{\mathcal{T}}=\mathfrak{d}_{\mathcal{T}^{*}}=1$, we have the following theorem.

Theorem 5.10. Let

$$
\mathrm{U}_{0}=\left(\begin{array}{cc}
-\mathrm{K} \mathcal{T}^{*} M & \mathrm{KD}_{\mathcal{T}} \\
\mathrm{D}_{\mathcal{T}} M & \mathcal{T}
\end{array}\right): \mathbb{C} \oplus \ell^{2}(\mathbb{Z}) \rightarrow \mathbb{C} \oplus \ell^{2}(\mathbb{Z}),
$$

be the prime unitary colligation with the characteristic function $\Theta_{\Delta_{0}}$. Let

$$
\mathrm{F}(\mu)=\left(\left(\mathbf{U}_{0}+\mu \mathrm{I}\right)\left(\mathbf{U}_{0}-\mu \mathrm{I}\right)^{-1} \overrightarrow{1}, \overrightarrow{1}\right)_{\mathbf{C} \oplus \ell^{2}(\mathbb{N})}, \mu \in \mathbf{D},
$$

where

$$
\mathrm{I}=\left[\begin{array}{ll}
1 & 0 \\
0 & \mathbf{1}
\end{array}\right]: \mathbb{C} \oplus \ell^{2}(\mathbb{Z}) \rightarrow \mathbb{C} \oplus \ell^{2}(\mathbb{Z}),
$$

is the operator in $\mathbb{C} \oplus \ell^{2}(\mathbb{Z})$ such that 1 is the scalar in $\mathbb{C}$ and $\mathbf{1}$ is the identity operator in $\ell^{2}(\mathbb{Z})$. Then

$$
F(\zeta)=\frac{1+\mu \overline{\Theta_{\Delta_{0}}(\mu)}}{1-\mu \overline{\Theta_{\Delta_{0}}(\mu)}}, \mu \in D \text {. }
$$

\subsection{Truncated CMV matrix}

Cantero et al. introduced five-diagonal matrix representation of a unitary operator with a single spectrum. Now we shall introduce this matrix representation and associated results. Note that one can find several papers including such a matrix representation [9-11, 24, 25].

Let $m$ be a probability measure $m$ on the unite circle. The function $F(z)$ defines a Carathéodory function:

$$
\mathrm{F}(z)=\mathrm{F}(z, \mathrm{~m}):=\int_{\mathbf{C}} \frac{\zeta+z}{\zeta-z} \mathrm{dm}(\zeta)=1+2 \sum_{n=1}^{\infty} \beta_{\mathrm{n}} z^{\mathrm{n}}, \beta_{\mathrm{n}}=\int_{\mathbf{C}} \zeta^{-\mathrm{n}} \mathrm{dm} .
$$


$F$ is analytic function in the disc $D$ and $\operatorname{ReF}>0, F(0)=1$. One defines the Schur function as follows

$$
f(z)=f(z, m):=\frac{1}{z} \frac{F(z)-1}{F(z)+1}, F(z)=\frac{1+z f(z)}{1-z f(z)} .
$$

$f(z)$ is an analytic function in the unit disc D with $\sup _{D}|f(z)| \leqslant 1$ [5]. There is a connection between probability measures, Carathéodory function and Schur function. Under this correspondence $\mathrm{m}$ is trivial if and only if the associated Schur function is a finite Blaschke product. Let $f=f_{0}$ be a Schur function and not a finite Blaschke product. Then we let

$$
f_{n+1}(z)=\frac{f_{n}(z)-\gamma_{n}}{z\left(1-\overline{\gamma_{n}} f_{n}(z)\right)}, \gamma_{n}=f_{n}(0)
$$

$\left\{f_{n}\right\}$ is an infinite sequence of Schur functions and neither of its terms is a finite Blaschke product. The numbers $\left\{\boldsymbol{\gamma}_{n}\right\}$ are called the Schur parameters

$$
\mathcal{S} f=\left\{\gamma_{0}, \gamma_{1}, \ldots\right\}
$$

If a Schur function $f$ is not a finite Blaschke product, the connection between the nontangental limit values $f(\zeta)$ and its Schur parameters $\left\{\gamma_{n}\right\}$ is given by

$$
\prod_{n=0}^{\infty}\left(1-\left|\gamma_{n}\right|^{2}\right)=\exp \left\{\int_{C} \ln \left(1-|f(\zeta)|^{2}\right) d m\right\} \text {. }
$$

Consequently,

$$
\sum_{n=0}^{\infty}\left|\gamma_{n}\right|^{2}=\infty \Leftrightarrow \ln \left(1-|\mathbf{f}(\zeta)|^{2}\right) \notin \mathrm{L}^{1}(\mathbf{C})
$$

Then we have the following.

Theorem 5.11. There exists a probability measure $\mathbf{m}$ on $\mathbf{C}$ such that $\mathcal{T}=(\mathcal{J}-\mathbf{i} \mathbf{1})(\mathcal{J}+\mathbf{i} \mathbf{1})^{-1}$ is unitary equivalent to the following operator

$$
\mathbb{T h}(\mu)=\mathrm{P}_{\mathfrak{K}}(\mu \mathrm{h}(\mu)), \mathrm{h} \in \mathfrak{K}:=\mathrm{L}^{2}(\mathbf{C}, \mathrm{dm}) \ominus \mathbb{C},
$$

where $\mathrm{P}_{\mathfrak{K}}$ is the orthogonal projection in $\mathrm{L}^{2}(\mathbf{C}, \mathrm{dm})$ onto $\mathfrak{K}$. The Schur function associated with $\mathrm{m}$ is the characteristic function $\Theta_{\mathcal{T}}(\mu)$ of $\mathcal{T}$ :

$$
f(\mu)=\Theta_{\mathcal{T}}(\mu)=\frac{\omega_{h}(\lambda)}{\omega_{\bar{h}}(\lambda)}, \mu=\frac{\lambda-i}{\lambda+i}, \operatorname{Im} \lambda>0 .
$$

If $\mathbf{m}$ is a nontrivial measure on $\mathbf{C}$, the monic orthogonal polynomials $\Phi_{\mathfrak{n}}(z, \mathbf{m})$ are uniquely determined by

$$
\Phi_{n}(z)=\prod_{j=1}^{n}\left(z-z_{n, j}\right), \int_{\mathbf{C}} \zeta^{-j} \Phi_{n}(\zeta) \mathrm{dm}=0, j=0,1, \ldots, n-1 .
$$

Therefore on the Hilbert space $\mathrm{L}^{2}(\mathbf{C}, \mathrm{dm}),\left(\Phi_{n}, \Phi_{\mathrm{m}}\right)=0, \mathrm{n} \neq \mathrm{m}$. Orthonormal polynomials $\phi_{\mathrm{n}}$ are of the form $\phi_{n}=\Phi_{n} /\left\|\Phi_{n}\right\|$.

(5.6) and the fact that the space of polynomials of degree at most $n$ have dimension $n+1$ imply

$$
\operatorname{deg}(P) \leqslant n, P \perp \zeta^{j}, j=0,1, \ldots, n-1 \Rightarrow P=c \Phi_{n}^{*} .
$$

Therefore $\Phi_{n+1}(z)-z \Phi_{n}(z)$ is of degree $n$ and orthogonal to $z^{j}, j=1,2, \ldots, n$, and

$$
\Phi_{n+1}(z)=z \Phi_{n}(z)-\bar{\alpha}_{n}(m) \Phi_{n}^{*}(z),
$$


where $\alpha_{n}(\mathbf{m})$ are complex numbers called Verblunsky coefficient. (5.7) is known as Szegö recurrences. For $z=0$, (5.7) implies

$$
\alpha_{\mathfrak{n}}(\mathbf{m})=\alpha_{n}=-\overline{\Phi_{n+1}(0)} .
$$

The inverse Szegö recurrences is

$$
z \Phi_{\mathfrak{n}}(z)=\rho_{\mathfrak{n}}^{-2}\left(\Phi_{n+1}(z)+\bar{\alpha}_{\mathfrak{n}} \Phi_{n}^{*}(z)\right)
$$

where

$$
\rho_{j}:=\sqrt{1-\left|\alpha_{j}\right|^{2}}, 0<\rho_{j} \leqslant 1,\left|\alpha_{j}\right|^{2}+\rho_{j}^{2}=1 .
$$

Then the norm $\left\|\Phi_{n}\right\|$ in $\mathrm{L}^{2}(\mathbf{C}, \mathrm{dm})$ may be determined as

$$
\left\|\Phi_{n}\right\|=\prod_{j=0}^{n-1} \rho_{j}, n=1,2, \ldots
$$

The CMV basis $\left\{\chi_{n}\right\}$ is obtained by orthonormalizing the sequence $1, \zeta, \zeta^{-1}, \zeta^{2}, \zeta^{-2}, \ldots$, and the matrix

$$
\mathcal{C}=\mathcal{C}(\mathbf{m})=\left\|c_{n, m}\right\|_{n, m=0}^{\infty}=\left\|\left(\zeta \chi_{m}, \chi_{n}\right)\right\|, m, n \in \mathbb{Z}_{+}
$$

is five-diagonal. The elements of $\left\{\chi_{n}\right\}$ may be expressed as follows:

$$
\chi_{2 n}(z)=z^{-n} \phi_{2 n}^{*}(z), \chi_{2 n+1}(z)=z^{-n} \phi_{2 n+1}^{*}(z), n \in \mathbb{Z}_{+} .
$$

Therefore one can find the matrix elements in terms of $\alpha^{\prime}$ s and $\rho^{\prime} s$ as

$$
\mathcal{C}=\mathcal{C}\left(\left\{\alpha_{n}\right\}\right)=\left(\begin{array}{cccccc}
\bar{\alpha}_{0} & \bar{\alpha}_{1} \rho_{0} & \rho_{1} \rho_{0} & 0 & 0 & \cdots \\
\rho_{0} & -\bar{\alpha}_{1} \alpha_{0} & -\rho_{1} \alpha_{0} & 0 & 0 & \cdots \\
0 & \bar{\alpha}_{2} \rho_{1} & -\bar{\alpha}_{2} \alpha_{1} & \bar{\alpha}_{3} \rho_{2} & \rho_{3} \rho_{2} & \cdots \\
0 & \rho_{2} \rho_{1} & -\rho_{2} \alpha_{1} & -\bar{\alpha}_{3} \alpha_{2} & -\rho_{3} \alpha_{2} & \cdots \\
0 & 0 & 0 & \bar{\alpha}_{4} \rho_{3} & -\bar{\alpha}_{4} \alpha_{3} & \cdots \\
\cdots & \cdots & \cdots & \cdots & \cdots & \cdots
\end{array}\right)
$$

Here $\alpha^{\prime}$ s are the Verblunsky coefficients and $\rho^{\prime}$ s are as given in (5.8). $\mathcal{C}\left(\left\{\alpha_{n}\right\}\right)$ is the matrix representation of the unitary operator of multiplication by $\zeta$ in $\mathrm{L}^{2}(\mathbf{C}, \mathrm{dm})$.

Finally we let the following matrix which is obtained from $\mathcal{C}$ by deleting the first row and the first column:

$$
\mathbb{T}=\mathbb{T}\left(\left\{\alpha_{n}\right\}\right)=\left(\begin{array}{ccccc}
-\bar{\alpha}_{1} \alpha_{0} & -\rho_{1} \alpha_{0} & 0 & 0 & \cdots \\
\bar{\alpha}_{2} \rho_{1} & -\bar{\alpha}_{2} \alpha_{1} & \bar{\alpha}_{3} \rho_{2} & \rho_{3} \rho_{2} & \cdots \\
\rho_{2} \rho_{1} & -\rho_{2} \alpha_{1} & -\bar{\alpha}_{3} \alpha_{2} & -\rho_{3} \alpha_{2} & \cdots \\
0 & 0 & \bar{\alpha}_{4} \rho_{3} & -\bar{\alpha}_{4} \alpha_{3} & \cdots \\
\cdots & \cdots & \cdots & \cdots & \cdots
\end{array}\right)
$$

Since $\mathfrak{d}_{\mathcal{T}}=\mathfrak{d}_{\mathcal{T}^{*}}=1$, following theorem follows immediately from the result of [5].

Theorem 5.12. $\mathcal{T}=(\mathcal{J}-\mathbf{i} \mathbf{1})(\mathcal{J}+\mathbf{i} \mathbf{1})^{-1}$ is unitary equivalent to the operator acting on $\ell^{2}(\mathbb{Z})$ determined by the truncated $C M V$ matrix $\mathbb{T}=\mathbb{T}\left(\left\{\alpha_{n}\right\}\right)$, where $\left\{\alpha_{n}\right\}$ are the Schur parameters of the characteristic function $\Theta_{\mathcal{T}}$ of $\mathcal{T}$.

\section{References}

[1] N. I. Akhiezer, The classical moment problem and some related questions in analysis, Translated by N. Kemmer, Hafner Publishing Co., New York, (1965). 1

[2] N. I. Akhiezer, I. M. Glazman, Theory of linear operators in Hilbert space, Vol. I, II, Translated from the third Russian edition by E. R. Dawson, Translation edited by W. N. Everitt, Monographs and Studies in Mathematics, Pitman (Advanced Publishing Program), Boston, Mass.-London, (1981). 1 
[3] B. P. Allakhverdiev, G. S. Guseinnov, On the spectral theory of second-order dissipative difference operators, (Russian); translated from Mat. Sb., 180 (1989), 101-118, Math. USSR-Sb., 66 (1990), 107-125. 2

[4] D. Alpay, A. Dijksma, J. Rovnyak, H. de Snoo, Schur functions, operator colligations, and reproducing kernel Pontryagin spaces, Operator Theory: Advances and Applications, Birkhäuser Verlag, Basel, (1997). 5.2

[5] Y. Arlinskiı̌, L. Golinskiŭ, E. Tsekanovskiŭ, Contractions with rank one defect operators and truncated CMV matrices, J. Funct. Anal., 254 (2008), 154-195. 1, 5.2, 5.8, 5.2, 5.2, 5.3, 5.3

[6] Y. Arlinskiĭ, E. Tsekanovskiü, Non-self-adjoint Jacobi matrices with a rank-one imaginary part, J. Funct. Anal., 241 (2006), 383-438. 1, 2

[7] J. Bognár, Indefinite inner product spaces, Ergebnisse der Mathematik und ihrer Grenzgebiete, Springer-Verlag, New York-Heidelberg, (1974). 3

[8] M. S. Brodskiǔ, Unitary operator colligations and their characteristic functions, (Russian); Uspekhi Mat. Nauk, 33 (1978), 141-168. 1, 5.2

[9] M. J. Cantero, L. Moral, L. Velázquez, Five-diagonal matrices and zeros of orthogonal polynomials on the unit circle, Linear Algebra Appl., 362 (2003), 29-56. 1, 5.3

[10] M. J. Cantero, L. Moral, L. Velázquez, Measures on the unit circle and unitary truncations of unitary operators, J. Approx. Theory, 139 (2006), 430-468.

[11] F. Gesztesy, M. Zinchenko, Weyl-Titchmarsh theory for CMV operators associated with orthogonal polynomials on the unit circle, J. Approx. Theory, 139 (2006), 172-213. 5.3

[12] I. C. Gohberg, M. G. Kreŭn, Introduction to the theory of linear non-self-adjoint operators, Translated from the Russian by A. Feinstein, Translations of Mathematical Monographs, American Mathematical Society, Providence, R.I., (1969). 1

[13] V. I. Gorbachuk, M. L. Gorbachuk, Boundary value problems for operator differential equations, Translated and revised from the 1984 Russian original, Mathematics and its Applications (Soviet Series), Kluwer Academic Publishers Group, Dordrecht, (1991). 2

[14] G. S. Guseinov, Completeness of the eigenvectors of a dissipative second order difference operator: dedicated to Lynn Erbe on the occasion of his 65th birthday, In honor of Professor Lynn Erbe. J. Difference Equ. Appl., 8 (2002), 321-331. 2

[15] E. Kreyszig, Introductory functional analysis with applications, John Wiley \& Sons, New York-London-Sydney, (1978). 2

[16] B. S. Nagy, C. Foiaş, Harmonic analysis of operators on Hilbert space, Translated from the French and revised NorthHolland Publishing Co., Amsterdam-London; American Elsevier Publishing Co., Inc., New York; Akadémiai Kiadó, Budapest, (1970). 1, 3, 3.4, 3

[17] B. S. Nagy, C. Foiaş, H. Bercovici, L. Kérchy, Harmonic analysis of operators on Hilbert space, Second edition, Revised and enlarged edition, Universitext, Springer, New York, (2010). 1, 3, 3, 5.1

[18] N. K. Nikolskiŭ, Treatise on the shift operator, Spectral function theory, With an appendix by S. V. Hruščev [S. V. Khrushchëv] and V. V. Peller, Translated from the Russian by Jaak Peetre, Grundlehren der Mathematischen Wissenschaften [Fundamental Principles of Mathematical Sciences], Springer-Verlag, Berlin, (1986). 3

[19] N. K. Nikolski, Operators, functions, and systems: an easy reading, Vol. 2, Model operators and systems, Translated from the French by Andreas Hartmann and revised by the author, Mathematical Surveys and Monographs, American Mathematical Society, Providence, RI, (2002). 1, 3.4, 3, 3, 3, 5.1, 5.1

[20] N. K. Nikolskiř, V. I. Vasjunin, Control subspaces of minimal dimension, unitary and model operators, J. Operator Theory, 10 (1983), 307-330. 5.1

[21] B. S. Pavlov, Dilation theory and spectral analysis of non-self-adjoint differential operators, (Russian); Mathematical programming and related questions, Proc. Seventh Winter School, Drogobych, (1974), Theory of operators in linear spaces (Russian), Central. Ékonom. Mat. Inst. Akad. Nauk SSSR, Moscow, (1976), 3-69. 1

[22] D. Sarason, On spectral sets having connected complement, Acta Sci. Math. (Szeged), 26 (1965), 289-299. 1

[23] Y.-M. Shi, H.-Q. Sun, Self-adjoint extensions for second-order symmetric linear difference equations, Linear Algebra Appl., 434 (2011), 903-930. 2

[24] B. Simon, Analogs of the m-function in the theory of orthogonal polynomials on the unit circle, J. Comput. Appl. Math., 171 (2004), 411-424. 5.3

[25] B. Simon, CMV matrices: five years after, J. Comput. Appl. Math., 208 (2007), 120-154. 5.3

[26] B. M. Solomyak, A functional model for dissipative operators, A coordinate-free approach. (Russian); translated from Zap. Nauchn. Sem. Leningrad. Otdel. Mat. Inst. Steklov. (LOMI), 178 (1989), Issled. Lineǐn. Oper. Teorii Funktsǐ̌., 18, 57-91, 184-185, J. Soviet Math., 61 (1992), 1981-2002. 1, 3, 3, 3, 4.1

[27] Z. Wang, H.-Y. Wu, Dissipative non-self-adjoint Sturm-Liouville operators and completeness of their eigenfunctions, J. Math. Anal. Appl., 394 (2012), 1-12. 2 